\title{
Epigenetic downregulation of STAT6 increases HIF-1a expression via mTOR/S6K/ S6, leading to enhanced hypoxic viability of glioma cells
}

Soo Jung Park ${ }^{1,2}$, Hyunmi Kim ${ }^{1,2}$, Se Hyuk Kim³ ${ }^{3}$ Eun-hye Joe ${ }^{1,2}$ and Ilo Jou ${ }^{1,2^{*}}$ (D)

\begin{abstract}
Multifunctional signal transducer and activator of transcription (STAT) proteins play important roles in cancer. Here, we have shown that STAT6 is epigenetically silenced in some cases of malignant glioblastoma, which facilitates cancer cell survival in a hypoxic microenvironment. This downregulation results from hypermethylation of $\mathrm{CpG}$ islands within the STAT6 promoter by DNA methyltransferases. STAT6 interacts with Rheb under hypoxia and inhibits mTOR/S6K/S6 signaling, in turn, inducing increased HIF-1a translation. STAT6 silencing and consequent tumor-promoting effects are additionally observed in glioma stem-like cells (GSC). Despite recent advances in cancer treatment, survival rates have shown little improvement. This is particularly true in the case of glioma, where multimodal treatment and precision medicine is needed. Our study supports the application of epigenetic restoration of STAT6 with the aid of DNA methyltransferase inhibitors, such as 5-aza-2-deoxycytidine, for treatment of STAT6-silenced gliomas.
\end{abstract}

Keywords: STAT6, DNA hypermethylation, Glioma, HIF-1a, mTOR, Rheb

\section{Introduction}

Epigenetic modifications comprising DNA methylations and histone modifications both qualitatively and quantitatively alter gene expression $[15,36]$. Accordingly, together with genetic mutations, epigenetic modifications can produce changes in physiologic functions that contribute to various diseases, including cancers. Recent genome-wide DNA methylation analyses and large-scale epigenetic profiling studies have revealed widespread distribution of epigenetic markers as well as frequent epigenetic alterations in a variety of human malignancies, thus establishing a causative role for an altered epigenome in carcinogenesis [1]. The predominant modification of mammalian DNA is cytosine methylation, followed by adenine and guanine methylation [17].

\footnotetext{
* Correspondence: jouilo@aumc.ac.kr

${ }^{1}$ Department of Pharmacology, School of Medicine, Ajou University, Suwon 16499, South Korea

${ }^{2}$ Chronic Inflammatory Disease Research Center, Ajou University, Suwon 16499, South Korea

Full list of author information is available at the end of the article
}

Methylation of cytosine bases in mammalian DNA has been primarily described in the context of CpG dinucleotides. Methylation of cytosine in the promoter region may repress expression of the corresponding gene by preventing the binding of specific transcription factors or attracting mediators of chromatin remodeling, such as histone-modifying enzymes or other repressors of gene expression.

An aberrant epigenome is a major risk factor in glioblastoma (GBM), the most malignant and lethal human brain tumor [27]. Importantly, hypermethylation of the DNA repair enzyme, $\mathrm{O}^{6}$-methylguanine-DNA methyltransferase (MGMT), which prevents repair of aberrant methylation, is a strong prognostic biomarker for the responsiveness of GBM patients to alkylating agents, including temozolomide [49]. Furthermore, The Cancer Genome Atlas (TCGA) project recently identified a subset of GBM tumors with cancer-specific CpG island hypermethylation, referred to as a glioma CpG island methylator phenotype (G-CIMP) [34] . These CIMP-positive tumors often exhibit characteristic molecular and clinicopathologic features, suggesting that 
CIMP represents a distinct carcinogenic pathway. The identification of CIMP could potentially lead to a better understanding of the molecular basis of tumorigenesis as well as improved treatment of cancer patients.

Accumulating evidence supports an important role of tissue hypoxia in cancer cell growth, neovascularization, invasion, resistance to chemo/radiotherapy and, ultimately, recurrence after treatment [13]. Adaptation to hypoxia allows for cancer progression in the hypoxic environment characteristic of the tumor core. A major mechanism that mediates adaptive responses to reduced $\mathrm{O}_{2}$ is activation of hypoxia-inducible factor-1 (HIF-1) [41]. HIF-1 levels and activity are primarily controlled by the availability of the oxygen-sensitive HIF- $1 \alpha$ subunits. In normoxia, prolyl hydroxylases (PHD) hydroxylate HIF-1 $\alpha$, triggering von Hippel-Lindau (pVHL)-mediated ubiquitination and proteasomal degradation. Conversely, hypoxia leads to inhibition of PHDs and stabilization of HIF-1 $\alpha$. In addition to protein stability, protein synthesis contributes to HIF- $1 \alpha$ accumulation in response to hypoxia. In this context, several studies have shown that mTOR, a key regulator of protein synthesis, plays a significant role in HIF- $1 \alpha$ accumulation under hypoxic conditions $[2,4,10]$. Relative to HIF-1 $\alpha$ regulation via protein degradation, the regulatory mechanisms governing translational processes are largely unknown.

High-level expression of HIF- $1 \alpha$ is positively correlated with tumor progression and poor prognosis in patients with GBM $[44,52]$. Small molecule- or siRNA-mediated inhibition of HIF-1 $\alpha$ can induce apoptosis in gliomas, sensitizing them to chemotherapeutic agents and impairing cancer cell migration and invasion in vitro under hypoxic conditions $[6,12]$. Hypoxia also provides a niche microenvironment that maintains glioma stem-like cells (GSCs) $[14,43]$, a phenomenon in which HIF-1 $\alpha$ plays a critical role [25]. Therefore, targeting HIF-1 $\alpha$ and its associated regulators and gene targets in glioma could lead to benefits for cancer patients.

Signal transducer and activator of transcription (STAT) proteins have important roles in many biological processes [45]; thus, not surprisingly, dysregulation of STAT signaling has been reported in many human diseases, including cancers. Dysregulation of STAT signaling can result from an increase in cytokine release and receptor activation, as well as upstream kinase hyperactivity [30]. In addition to protein activity, the amount of STAT protein is important in tumorigenesis. Gain-offunction mutations in STAT3 and STAT5 have been reported in leukemia and lymphoma [20, 21, 33]. Epigenetically silenced STAT may also serve a tumor-promoting function. For example, STAT1 and STAT5A genes are silenced by DNA methylation in squamous cell carcinoma of the head and neck (SCCHN) and NPM1-ALK-expressing lymphomas, respectively $[51,53]$. Although aberrant STAT signaling has been linked to diverse aspects of GBM tumor progression, invasion and GSC maintenance $[3,26]$, the contribution of STAT gene dysregulation to tumor pathology, particularly at the epigenetic level, is unclear.

Despite recent clinical trials of targeted therapies, further advances in therapeutic strategies have stalled, possibly reflecting the complex heterogeneity of cancer cells. In this study, we demonstrated that STAT6, an important signaling molecule in adaptive immunity, is frequently silenced in gliomas where hypoxia is a prominent feature. Based on the findings, we propose that STAT6 downregulation resulting from DNA methyltransferase (DNMT)-mediated hypermethylation of promoter CpG islands facilitates accumulation of HIF- $1 \alpha$ through mTOR activation in hypoxia and consequent enhancement of glioma cell survival. mTOR activation via STAT6 knockdown is achieved through suppression of direct interactions between STAT6 and Rheb that inhibit HIF-1 $\alpha$ translation. Moreover, STAT6 silencing and resulting tumorpromoting effects were consistently observed in glioma stem-like cells. Recent developments in application of precision medicine to cancer treatment support the application of epigenetic restoration of STAT6 via DNA methyltransferase inhibitors (DNMTi) as a therapeutic strategy for STAT6-silenced gliomas.

\section{Materials and methods \\ Reagents and antibodies}

Cycloheximide, 5-aza-2'-deoxycytidine (5-Aza), trichostatin A (TSA), and rapamycin were purchased from Sigma-Aldrich (St. Louis, MO, USA). The indicated primary antibodies against the following proteins were used in this study: STAT1/2/3/4/5/6 (\#9172/4594/ 12640/5097/9363/9362), DNMT1/3a/3b (\#5119/3598/ 67259), cleaved-caspase 3/7 (\#9664/9491), cleaved PARP (\#5625), S6K (\#2708), phospho-S6K (T389) (\#9205), S6 (\#2217), phospho-S6 (Ser235/236) (\#2211), mTOR (\#2972), 4E-BP1 (\#9644), phospho-4E-BP1 (T70) (\#9455), eIF4E (\#2067), phospho-eIF4E (S209) (\#9741), eIF4G (\#2469), Rheb (\#13879), TSC2 (\#4308), p-TSC2 (T1462) (\#3617), p-TSC2 (S1387) (\#5584), ICAM1 (\#4915), JAK2 (\#3230), NFATC2 (\#4389) and Myc taq (\#2278) (Cell Signaling Technology, Danvers, MA, USA); anti-HIF-1 $\alpha$ (610958) (BD Biosciences, San Jose, CA, USA); and anti-actin (sc-1616) and GAPDH (sc-48,167) (Santa Cruz Biotechnology, Santa Cruz, CA, USA). Secondary antibodies used were anti-goat IgG HRP (81-1620; Invitrogen, Carlsbad, CA, USA), antimouse IgG HRP (G-21040; Invitrogen), and anti-rabbit IgG HRP (111-035-003; Jackson Laboratories, Bar Harbor, ME, USA). 


\section{Cell lines and tumor samples}

The human glioblastoma cell lines U87MG and U373MG were obtained from the Korean Cell Line Bank (Seoul, Korea) and U251 and LN229 were kindly provided by Dr. Hee Young Kim (Seoul National University, Seoul, Republic of Korea). The cancer cell lines were routinely grown in Dulbecco's Modified Eagle Medium (DMEM; Sigma-Aldrich) containing 10\% fetal bovine serum (FBS; Gemini, West Sacramento, CA, USA) and $0.1 \%$ antibiotic-antimycotic solution (Gibco, Carlsbad, CA, USA).

Fetal normal human astrocytes (NHA) were purchased from ABM (Richmond, BC, Canada) and were cultured according to manufacturer's direction in Prigrow $\mathrm{X}$ Series Medium (ABM). Glioma stem-like cells (GSCs) were established from freshly resected tumors and were cultured in neurobasal media (Gibco) supplemented with N2 (Gibco) and B27 (Invitrogen). Cultures were supplemented with $20 \mathrm{ng} / \mathrm{mL}$ of epidermal growth factor (EGF) (Invitrogen) and basic fibroblast growth factor (bFGF) (Millipore, Billerica, MA, USA) every 2-3 days.

All cells were incubated at $37^{\circ} \mathrm{C}$ in a humidified atmosphere of $95 \%$ air and $5 \% \mathrm{CO}_{2}$. For hypoxia treatment, cells were incubated in an oxygen control hypoxia chamber (Coy Laboratory Products, Grass Lake, MI, USA) at $37^{\circ} \mathrm{C}$ in a humidified $5 \% \mathrm{CO}_{2}$ environment, with the balance provided by $\mathrm{N}_{2}$.

Following informed consent, glioma and normal brain tissues were obtained from patients undergoing surgery at the Ajou University Hospital in accordance with Institutional Review Boards protocols. The samples were snap-frozen in liquid nitrogen and stored at $-80^{\circ} \mathrm{C}$ until analysis. Detailed information of patients is provided in Additional file 2: Table S1.

\section{Immunoblotting}

Cells were lysed in RIPA buffer (50 mM Tris- $\mathrm{HCl}, 1 \%$ NP-40, $0.25 \%$ sodium deoxycholate, $150 \mathrm{mM} \mathrm{NaCl}, 1$ mM EDTA) supplemented with protease inhibitor cocktail and phosphatase inhibitor cocktail. The protein concentration in lysates was determined using the Bio-Rad protein assay (Bio-Rad Laboratories, Hercules, CA, USA). Proteins were separated by sodium dodecyl sulfate-polyacrylamide gel electrophoresis (SDS-PAGE) and transferred to nitrocellulose membranes. Primary antibodies were applied overnight at $4{ }^{\circ} \mathrm{C}$. Peroxidaseconjugated secondary antibodies were applied at room temperature for $1 \mathrm{~h}$, and immunoreactive protein were visualized using an enhanced chemiluminescence detection kit (WESTSAVE Gold; AbFrontier, Seoul, Korea), followed by exposure to X-ray film. Band intensities were quantified using ImageJ software (National Institutes of Health, Bethesda, MD, USA).

\section{Immunohistochemistry}

A tissue microarray (TMA) containing glioblastoma and normal brain samples (GL806; US Biomax,Rockville, MD, USA) was used for high throughput analysis of gene expression. The TMA contains 35 cases of glioblastoma and 5 normal brain tissues, represented as duplicate cores per case. Tissue microarray slides were deparaffinized in xylene followed by rehydration. For antigen retrieval, slides were boiled in $10 \mathrm{mM}$ citric acid buffer (pH 6.0) for $20 \mathrm{~min}$, followed by blocking of endogenous peroxidase by incubating in $0.3 \% \mathrm{H}_{2} \mathrm{O}_{2}$ in methanol for $30 \mathrm{~min}$. Slides were blocked by incubating for $1 \mathrm{~h}$ in $10 \%$ normal goat serum, and then incubated overnight at $4{ }^{\circ} \mathrm{C}$ with a primary antibody against STAT6 (1:100; Abcam, Cambridge, MA, USA) and then with biotinylated goat anti-rabbit IgG secondary antibody (1:400) for $1 \mathrm{~h}$. The antigen-antibody reaction was visualized using the Vector SG peroxidase substrate in a Vectastain Elite ABC kit (Vector Laboratories, Burlingame, CA, USA). Slides were counterstained with Harris hematoxylin solution (Sigma), subjected to a dehydration process, then briefly washed in xylene and mounted with VectaMount Permanent Mounting Medium (Vector Laboratories). For the quantitative analysis, a Histo score ( $\mathrm{H}$ score) was calculated based on the staining intensity and percentage of stained cells. The intensity score was defined as follows: 1, weak staining; 2, intermediate staining; 3, strong staining. The $\mathrm{H}$ score was determined by the formula: $3 \times$ percentage of strong staining $+2 \times$ percentage of moderate staining + percentage of weak staining, giving a range of 0 to 300 for the $\mathrm{H}$ scores.

\section{Reverse transcription and quantitative real-time PCR analysis}

Total RNA was isolated using RNAiso Plus (TaKaRa, Otsu, Japan), and cDNA was synthesized using avian myeloblastosis virus reverse transcriptase (New England Biolabs) and oligo (dT) primers (Promega, Madison, WI, USA), according to the manufacturer's instructions. For qPCR, amplification reactions were performed using a Thermal Cycler Dice Real-Time System (TaKaRa) with SYBR Premix Ex Taq master mix (TaKaRa) according to the manufacturer's instructions. The primers used for RT-qPCR (Bioneer, Daejeon, Korea) are described in Additional file 2: Table S2.

\section{Methylation-specific PCR and pyrosequencing of bisulfate-converted genomic DNA}

The methylation status of STAT6 promoter CpG islands was analyzed using methylation-specific PCR (MSP) and pyrosequencing. Genomic DNA (gDNA) was extracted from human glioma and normal brain tissue and U373MG and U87MG cell lines using DNeasy Blood \& Tissue kits (Qiagen Hilden, Germany). Bisulfite conversion of gDNA was performed using the EpiTect Bisulfite Kit (Qiagen) and 


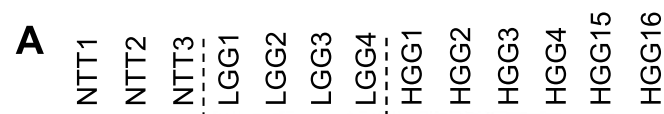
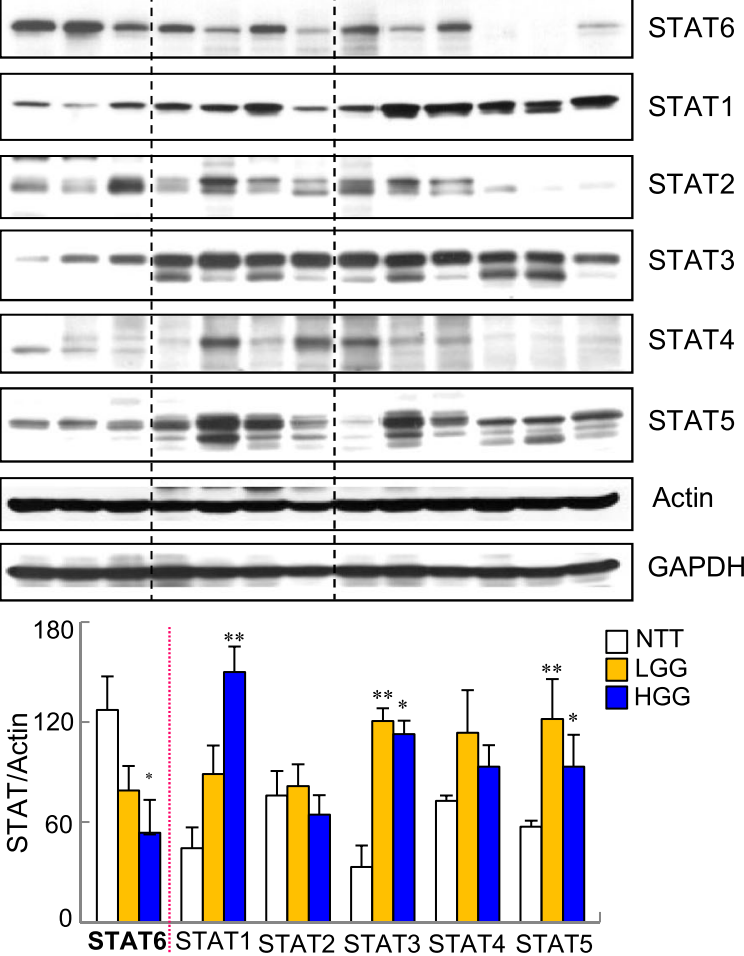

C

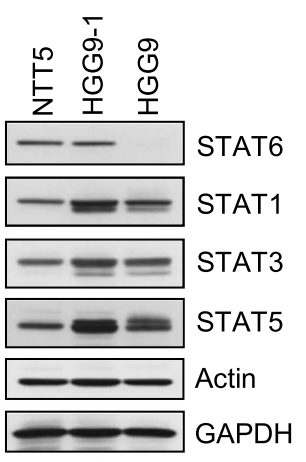

\section{B STAT6/Hematoxyin}
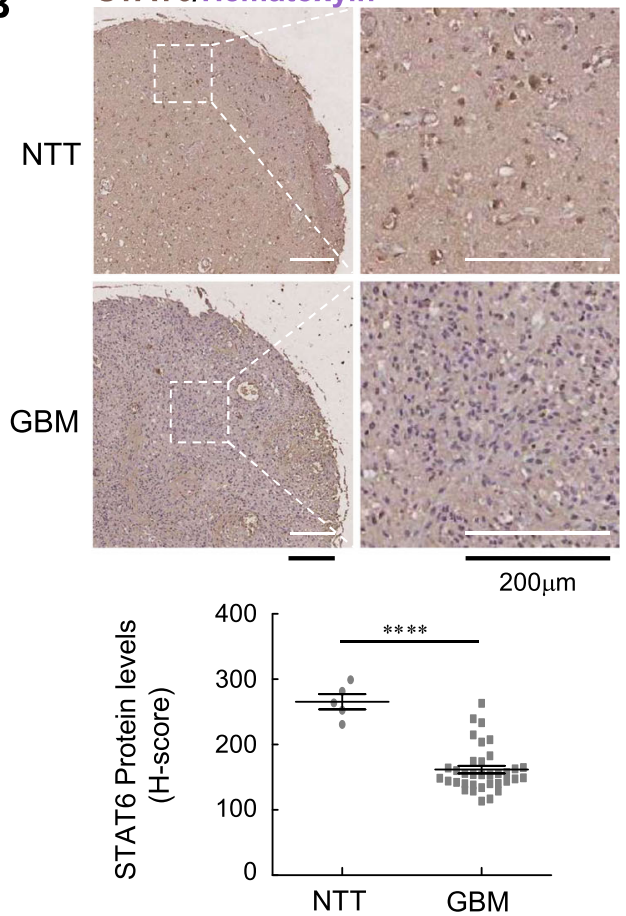

$\mathbf{E}$

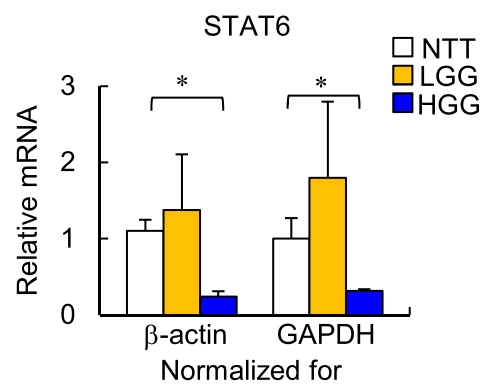

$\mathbf{F}$

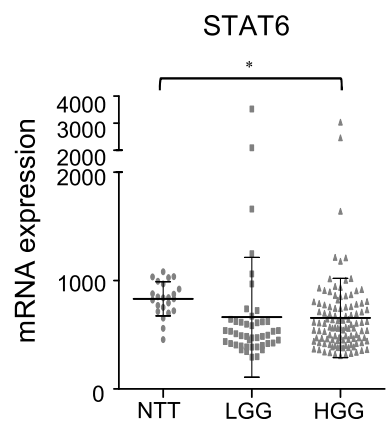

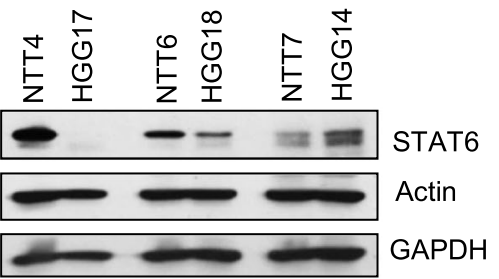

D

G

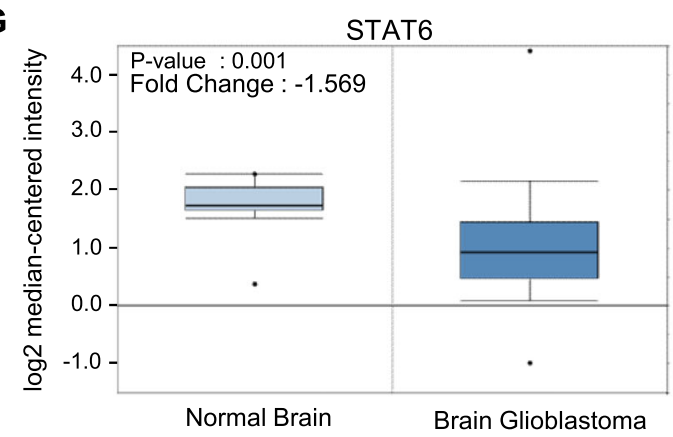

Fig. 1 (See legend on next page.) 
(See figure on previous page.)

Fig. 1 STAT6 is downregulated in glioma. a Immunoblot of the indicated STATs in non-tumor brain tissue (NTT), low-grade glioma tissue (LGG), and high-grade glioma tissue (HGG) (top) and summary data (bottom) showing actin-normalized levels of STATs. b Representative immunohistochemical staining of STAT6 in normal and GBM tissue (top) and summary data (bottom) showing the quantification (H-score, see Materials and Methods) of STAT6 staining. $\mathbf{c}$ Immunoblot of the indicated STATs in paired normal (NTT5), primary (HGG9-1) and recurrent GBM (HGG9) samples. N, normal; T, tumor. d Immunoblot of STAT6 in GBM (HGG17 and HGG18) and anaplastic oligodendroglioma (HGG14) and paired normal tissues from each. NTT4, HGG17-paired; NTT6, HGG18-paired; NTT7, HGG14-paired. e STAT6 mRNA levels in NTT $(n=3), \mathrm{LGG}(n=3)$ and HGG $(n=3)$, determined by RT-qPCR. Transcript levels were normalized to GAPDH or actin levels. $\mathbf{f}$ Expression of STAT6 from GEO data set GDS1962 containing 23 non-tumor (NTT), 45 grade II (LGG), and 31 and 81 grade III and IV (HGG) samples $\left({ }^{*} p<0.05\right.$, ${ }^{* *} p<0.005$, and ${ }^{* * *} p<$ 0.0005 vs. NTT). $\mathbf{g}$ Expression of STAT6 from Oncomine data set TCGA brain consisting of normal brain $(n=10)$ and brain glioblastoma $(n=542)$ samples

then the converted DNA was amplified with primers specific to methylated or unmethylated DNA using an EpiTect MSP Kit (Qiagen), according to the manufacturer's instructions. The methylation-specific primer sequences in STAT6 promoter CpG islands were 5'-GCG TCG AGT TAA TTT TTT TC-3' (forward) and 5'-CGC TTA ATA ACC TAA ACT CGC-3' (reverse); the unmethylation-specific primer sequences were 5'-GGT GTT GAG TTA ATT TTT TTT3' (forward) and 5'-CCA CTT AAT AAC CTA AAC TCA C-3' (reverse). After the reaction was complete, products were analyzed by electrophoresis on a $2.5 \%$ agarose gel.

Pyrosequencing was performed by Genomictree (Daejeon, South Korea) using standard protocols. Briefly, bisulfite-modified gDNA was prepared using an EZ DNA Methylation-Lighting kit (Zymo Research, USA), and then converted gDNA was amplified by PCR. The reaction conditions were as follows: denaturation at $95^{\circ} \mathrm{C}$ for $10 \mathrm{~min}$, followed by 45 cycles of $95^{\circ} \mathrm{C}$ for $30 \mathrm{~s}, 58^{\circ} \mathrm{C}$ for $30 \mathrm{~s}, 72^{\circ} \mathrm{C}$ for $30 \mathrm{~s}$, and a final extension at $72{ }^{\circ} \mathrm{C}$ for $5 \mathrm{~min}$. Pyrosequencing was performed on a PyroMark ID system using a Pyro Gold reagents kit (Qiagen) according to the manufacturer's instruction without further optimization. The methylation percentage was calculated based on the average degree of methylation at 2-5 CpG sites formulated in pyrosequencing. Each primer was designed using Pyrosequencing Assay Design Software v2.0 (Qiagen). Primer sequences are listed in Additional file 2: Table S3.

\section{Knockdown and overexpression experiments}

For knockdown of specific proteins, cells were transfected for $72 \mathrm{~h}$ with siRNA (final RNA concentration, $25 \mathrm{nM}$ ) using DharmaFECT 2 reagents (Dharmacon), according to the manufacturer's instructions. SMART pool siRNA against STAT6 (L-006690-00) (Dharmacon Thermo Scientific) and non-targeting control siRNAs (D-001810-10) were purchased from Dharmacon (Lafayette, CO, USA); siRNA against DNMT1 (1786-1), DNMT3b (1789-1), HIF- $1 \alpha(3091-1)$ and a corresponding negative control (SN-1002) were purchased from Bioneer (Korea). siRNA against DNMT3a was chemically synthesized by Bioneer (Korea). The siRNA sequences are as follows: $5^{\prime}$-CAG GAG AUG AUG UCC AAC CC-3' (sense) and 5'-GGG UUG GAC AUC AUC UCC UG-3' (antisense).
STAT6 was overexpressed by transiently transfecting U373MG cells with pCMV6-Myc-DDK-STAT6 (RC210 065; Origene Technologies) or mock control vector (PS100001; Origene Technologies) using TurboFectin 8.0 (OriGene Technologies, Inc.) according to the manufacturer's recommendations. All assays were performed at least $48 \mathrm{~h}$ after transfection. STAT6 deletion mutants were generated from human STAT6 cDNA (RC210065, Origene) by PCR amplification using the appropriate primers. STAT6 deletion mutants $(\Delta 1-260$ (d1), $\Delta 261-430$ (d2), $\Delta 431-522(\mathrm{~d} 3)$, and $\Delta 523-622(\mathrm{~d} 4))$ were cloned into the pCMV6-entry vector (PS100001, Origene). Primer sequences are listed in Additional file 2: Table S4.

\section{Immunofluorescence and confocal microscopy}

U373MG cells cultured on poly-d-lysine-coated coverslips were fixed with $4 \%(\mathrm{v} / \mathrm{v})$ paraformaldehyde at $4{ }^{\circ} \mathrm{C}$ for $15 \mathrm{~min}$ and permeabilized by incubating for $5 \mathrm{~min}$ in phosphate-buffered saline (PBS) containing $0.1 \%$ Triton $\mathrm{X}-100$. Fixed cells were incubated with anti-STAT6 (611,290, BD Biosciences) and HIF-1 $\alpha$ (ab51608, Abcam) antibodies at $4{ }^{\circ} \mathrm{C}$ overnight, followed by incubation with Alexa Flour 488- or Alexa Flour 543-conjugated secondary antibodies (Molecular Probes, Eugene, OR, USA) for $2 \mathrm{~h}$. Cells were mounted with DAPI-containing mounting solution and observed under a confocal microscope (LSM510; Carl Zeiss, Jena, Germany).

\section{Nascent HIF-1a synthesis assay}

Newly synthesized proteins were detected using the Click-IT method (Life Technologies), according to the manufacturer's instructions. Briefly MOCK- or fulllength STAT6-transfected U373MG cells or control siRNA- or STAT6 siRNA-transfected U87MG cells were exposed to hypoxia for $18 \mathrm{~h}$. Cells were subsequently washed with PBS, incubated in methionine-free DMEM for $1 \mathrm{~h}$, then pulsed with $50 \mu \mathrm{M}$ of the methionine analog, L-azidohomoalanine (AHA) (Cat \# C10102; Life Technologies), for $2 \mathrm{~h}$, after which cells were harvested. AHA-incorporated proteins were labeled with biotin using a Click-iT Biotin Protein Analysis Detection Kit (Cat\# C33372; Invitrogen). Biotin-labeled proteins were pulled down using a streptavidin-agarose bead slurry 


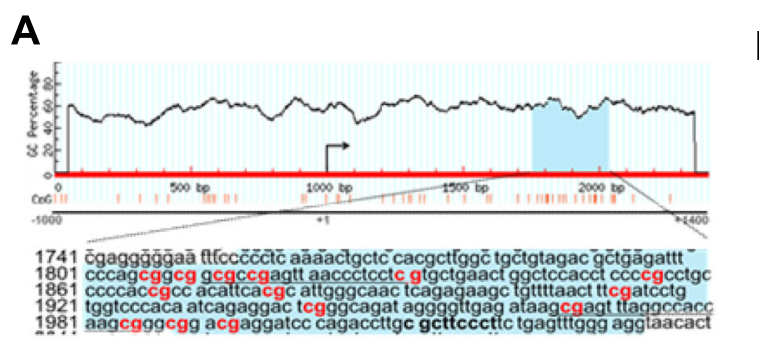

D

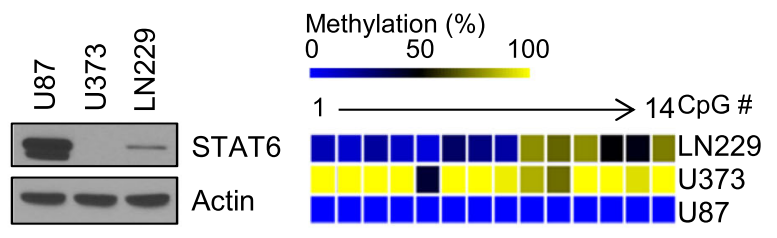

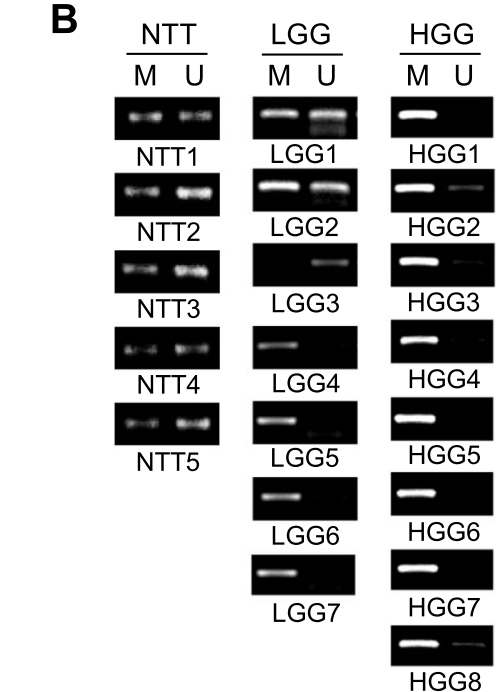

C

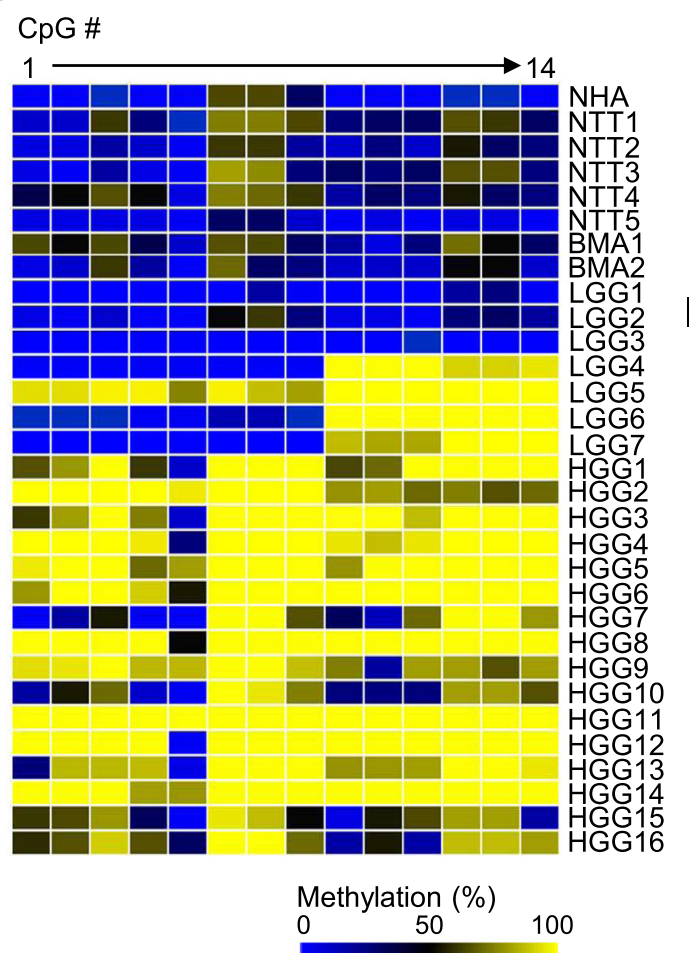

E

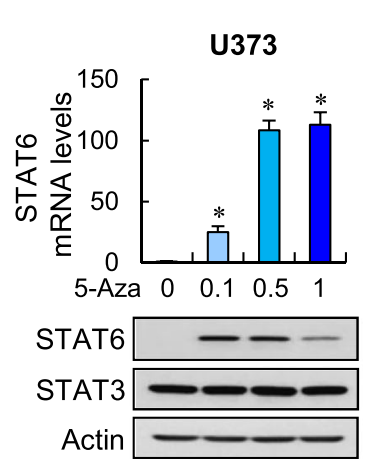

G

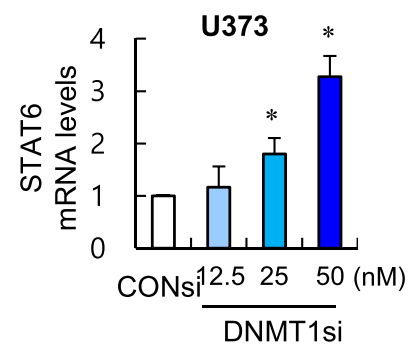

I

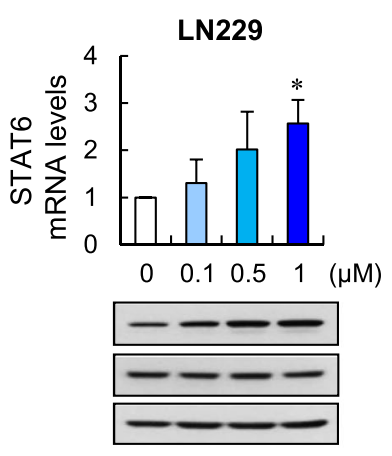

F U373

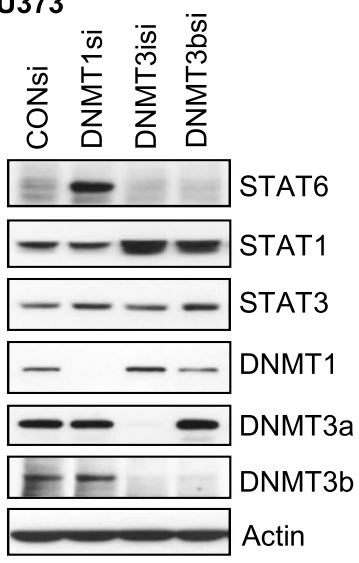

H U373

$$
\sum_{0}^{\bar{m}} \frac{\text { DNMT1si }}{6.2512 .52550(n M)}
$$

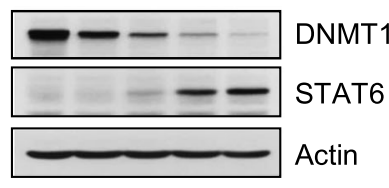

LN229
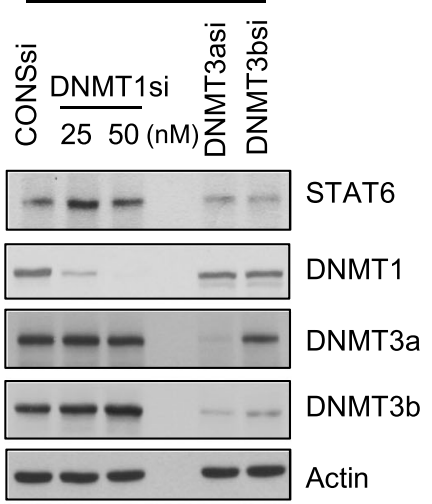

Fig. 2 (See legend on next page.) 
(See figure on previous page.)

Fig. 2 DNMT1-dependent DNA methylation inhibits STAT6 expression. a Distribution of CpG islands in the -1000 to + 1400 STAT6 promoter region. Two underlined sequences show the locations of MSP-PCR primers; 14 analyzed CpG sites are shown in bold red font. b MSP of STAT6 promoter CpG islands in non-tumor brain tissue (NTT), low-grade glioma tissue (LGG), and high-grade glioma tissue (HGG). M, methylated product; $U$, unmethylated product. c Bisulfite sequencing of $14 \mathrm{CpG}$ sites in STAT6 promoter CpG islands in normal human astrocytes (NHAs), NTT, brain metastatic adenocarcinoma (BMA), LGG, and HGG tissue. Each square represents $1 \mathrm{CpG}$ analyzed, and colors indicate the percentage of methylation. $\mathbf{d}$ Immunoblot of STAT6 (left) and bisulfite sequencing (right) of STAT6 promoter CpG islands in the indicated GBM cell lines. e RTqPCR of STAT6 (top) and immunoblot of indicated proteins (bottom) in U373MG cells treated with indicated doses of 5-Aza for $72 \mathrm{~h}$. Results are presented as means \pm SD (error bars) of three independent experiments $\left({ }^{*} p<0.05\right.$ and ${ }^{* *} p<0.01$ vs. untreated cells). $\mathbf{f}$ Immunoblot of the indicated STATs and DNMTs in U373MG cells transfected with the indicated DNMT siRNA (DNMTsi) or control siRNA (CONsi). $\mathbf{g}, \mathbf{h}$ U373MG cells transfected with CONsi or indicated doses of DNMT1si, followed by quantification of STAT6 mRNA by RT-qPCR ( $\mathbf{g})$ and immunoblotting for DNMT1 and STAT6 in cells from $\mathrm{g}(\mathbf{h})$. Results are presented as means \pm SD (error bars) of three independent experiments ${ }^{*} p<0.05$ vs. CONsitransfected cells). i RT-qPCR of STAT6 in LN229 cells treated with indicated doses of 5-Aza for $72 \mathrm{~h}$ (left) and immunoblot of STAT6 and the indicated DNMTs in LN229 cells transfected with the indicated DNMT siRNA or control siRNA (right). Results are presented as means \pm SD (error bars) of three independent experiments $\left({ }^{*} p<0.05\right.$ and ${ }^{* *} p<0.01$ vs. untreated cells)

(Pierce, Rockford, IL, USA) and analyzed by immunoblotting using an anti-HIF- $1 \alpha$ antibody.

\section{Immunoprecipitation}

Cells were lysed in CHAPS buffer (40 mM HEPES pH 7.4, $120 \mathrm{mM} \mathrm{NaCl}, 1 \mathrm{mM}$ EDTA, 0.3\% CHAPS) supplemented with protease inhibitor cocktail and phosphatase inhibitor cocktail. Lysates $(300 \mu \mathrm{g})$ were incubated with antibody $(1 \mu \mathrm{g}$ of anti-mTOR, STAT6, eIF4E or isotype control antibodies) at $4{ }^{\circ} \mathrm{C}$ overnight. Magnetic protein $\mathrm{G}$ Dynabeads (Invitrogen) were then added, and tubes were rotated for an additional $2 \mathrm{~h}$. After washing five times with lysis buffer, proteins were eluted from beads with 2X Laemmli buffer at $95^{\circ} \mathrm{C}$ for $10 \mathrm{~min}$, followed by resolution by SDS-PAGE.

\section{Cell viability assay}

Cell viability was assessed using the EZ-Cytox Cell Viability Assay kit (Daeil Lab Service, Seoul, Korea). Control siRNAor STAT6 siRNA-transfected U87MG cells were exposed to hypoxia for 5 days. The Ez-Cytox Kit reagent was then added to the medium and cells were incubated at $37^{\circ} \mathrm{C}$ for $2 \mathrm{~h}$. The plate was read on an iMark microplate absorbance reader (Bio-Rad) at a wavelength of $450 \mathrm{~nm}$.

Cell viability was also assessed using the LIVE/DEAD ${ }^{\mathrm{mm}}$ Viability/Cytotoxicity Kit (Molecular Probes). Control siRNA- or STAT6 siRNA-transfected U87MG cells exposed to hypoxia for 5 days were washed twice with PBS and then incubated in presence of $0.5 \mu \mathrm{L} / \mathrm{mL}$ CalceinAM (Live/green) and $2 \mu \mathrm{L} / \mathrm{mL}$ EthD-1(Dead/green) for $20 \mathrm{~min}$ at room temperature in dark. Fluorescence images were collected using a fluorescence microscope (Zeiss Axiovert 200 M; Carl Zeiss).

\section{Flow cytometry}

U373MG cells were stained with propidium iodide (PI) (Sigma-Aldrich) for cell cycle analysis, or with PI and annexin V-FITC (BD Biosciences) for detection of apoptosis. The distribution of cells in the different phases of the cell cycle (based on differences in DNA content) and apoptosispositive cells were determined by flow cytometry using a FACSCalibur flow cytometer (BD Biosciences).

\section{CDNA microarray}

U373MG cells were transfected with control siRNA or STAT6 siRNA for 2 days. One day after transfection, cells were incubated with or without 5-Aza ( $300 \mathrm{nM})$ for 2 days and then the medium was replaced with fresh medium (without drug). Total RNA was extracted from cells on day 6 after drug withdrawal using the TRIzol reagent (Invitrogen). RNA samples were converted to labeled singlestranded DNA and hybridized to the GeneChip Human 2.0 ST array (Affymetrix). After hybridization, the chips were stained and washed in a Genechip Fluidics Station 450 and scanned on a GCS3000 Scanner (Affymetrix). Array data export, processing, and analysis were performed using Affymetrix GeneChip Command Console software. Raw data were extracted automatically in the Affymetrix data extraction protocol using the provided Affymetrix GeneChip Command Console Software (AGCC). After importing CEL files, the data were summarized and normalized using the robust multi-average (RMA) method implemented in Affymetrix Expression Console Software (EC). The results of gene-level RMA analysis were exported, and differentially expressed genes (DEGs) were analyzed by performing a comparative analysis of fold-change between test sample and control sample. A hierarchical cluster analysis was performed on the DEG set using complete linkage and Euclidean distance as a measure of similarity. Gene-Enrichment and Functional Annotation analyses of the significant probe list were performed using Gene Ontology (http://geneontology.org/). The R statistical language v. 3.1.2. (www.r-project.org) was used for all statistical tests and visualization of differentially expressed genes.

\section{ELISA}

Control siRNA- or STAT6 siRNA-transfected U373MG cells were treated with or without 5-Aza, after which the 


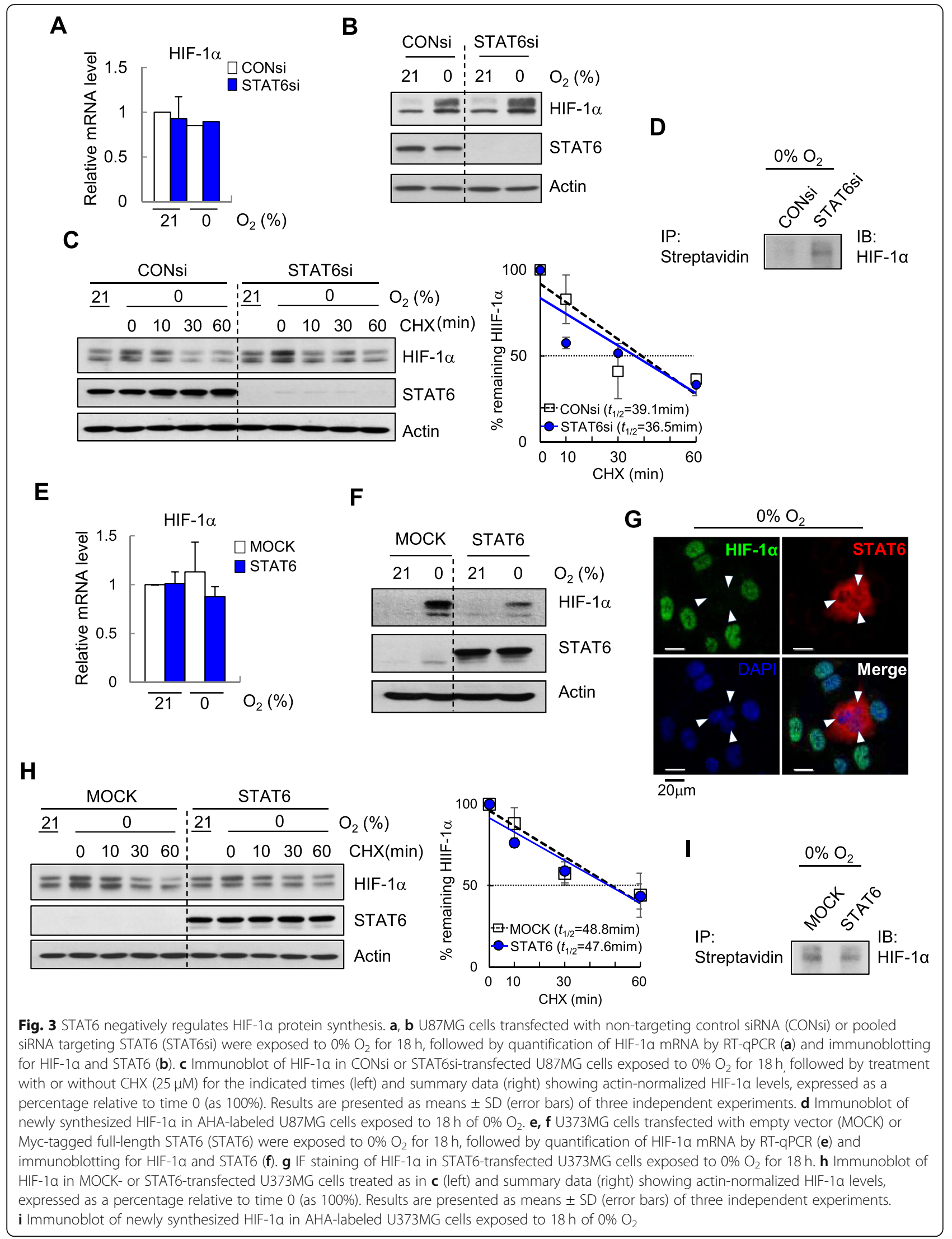


A

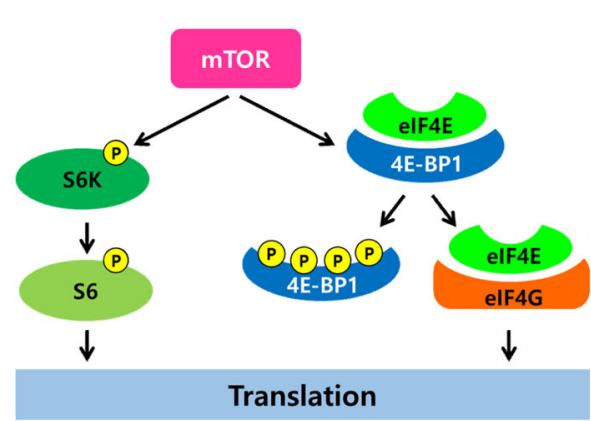

B

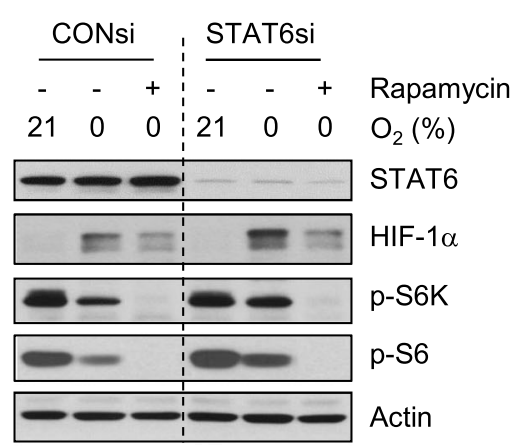

C

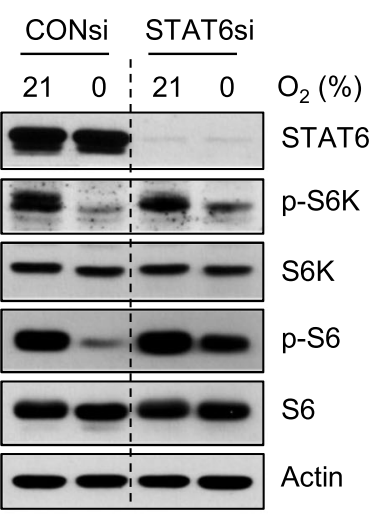

$\square$ CONsi $\square$ STAT6si

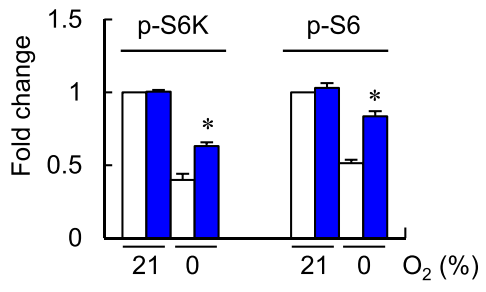

F

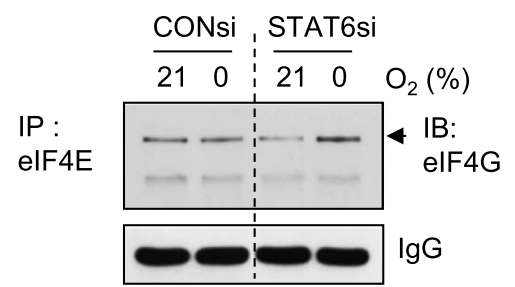

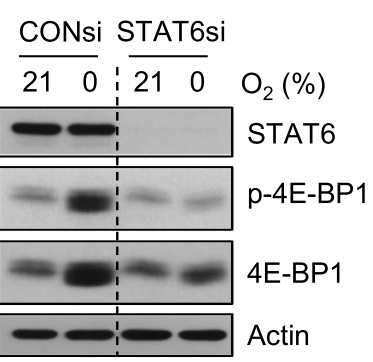

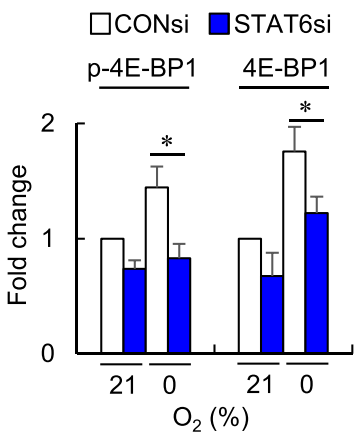

E
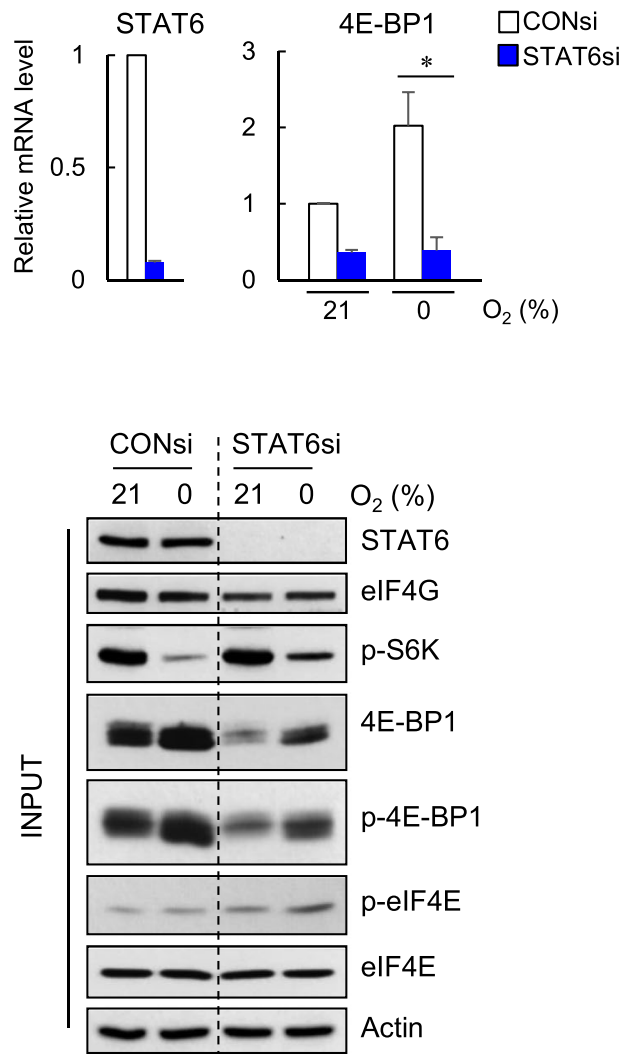

Fig. 4 (See legend on next page.) 
(See figure on previous page.)

Fig. 4 STAT6 negatively regulates mTOR signaling. a Schematic of downstream mTOR signaling. b Immunoblot of HIF-1a, p-S6K, p-S6 and STAT6 in CONsi or STAT6si -transfected U87MG cells exposed to $0 \% \mathrm{O}_{2}$ for $18 \mathrm{~h}$, with or without rapamycin (1 nM). c Immunoblot of the indicated proteins (top) and summary data (bottom) showing actin-normalized p-S6K and p-S6 levels expressed as fold-changes relative to that in CONsitransfected cells exposed to $21 \% \mathrm{O}_{2}$ in siRNA-transfected U87MG cells exposed to $0 \% \mathrm{O}_{2}$ for $18 \mathrm{~h}$. Results are presented as means $\pm \mathrm{SD}$ (error bars) of three independent experiments $\left({ }^{*} p<0.05\right)$. $\mathbf{d}$ Immunoblot of the indicated proteins (left) and summary data (right) showing actinnormalized p-4E-BP1 and 4E-BP1 levels expressed as fold-changes relative to that in CONsi-transfected cells exposed to $21 \% \mathrm{O}_{2}$ in siRNAtransfected U87MG cells exposed to $0 \% \mathrm{O}_{2}$ for $18 \mathrm{~h}$. Results are presented as means $\pm \mathrm{SD}$ (error bars) of three independent experiments ${ }^{*} p<$ 0.05). e RT-qPCR of $4 E-B P 1$ in siRNA-transfected U87MG cells exposed to $0 \% \mathrm{O}_{2}$ for $18 \mathrm{~h}$. Results are presented as means \pm SD (error bars) of three independent experiments $\left({ }^{*} p<0.05\right)$. $\mathbf{I}$ Immunoblot of elF4G in elF4E IPs from U87MG cells transfected with CONsi or STAT6si and exposed to 0\% $\mathrm{O}_{2}$ for $10 \mathrm{~h}$

concentrations of CCL2, CCL20, IL-8, and IL-1 in culture supernatants were detected using commercial ELISA kits, according to the manufacturer's instructions. Optical density was determined on an iMark microplate absorbance reader (Bio-Rad) at a wavelength of $450 \mathrm{~nm}$. Human CCL2 (DCP00), CCL20/MIP-3 $\alpha$ (DM3A00), IL8/CXCL8 (D8000C), and IL-1 $\beta /$ IL-1F2 (DLB50) ELISA Kit were purchased from R\&D Systems (Minneapolis, MN, USA).

\section{Statistical analysis}

GraphPad Prism 5.0 was used for statistical analyses. Differences between two groups were analyzed using an unpaired two-tailed Student's t-test. All results are presented as means and standard deviation (S.D.) of at least three independent experiments. The results were considered statistically significant at $p$-values $<0.05$.

\section{Results}

\section{STAT6 expression is downregulated in human glioma}

To explore the function and significance of STAT proteins in gliomas, we first examined the expression levels of individual STAT proteins in human glioma specimens. Western blot analyses demonstrated that, whereas STAT2 and STAT4 showed no prominent or consistent changes in expression, STAT1, STAT3 and STAT5 expression were increased in glioma tissues compared with neighboring non-tumor tissue. In contrast, STAT6 expression, which was apparent in non-tumor tissue, was decreased in glioma tissues, exhibiting an inverse relationship with tumor grade (Fig. 1a). Although it is well known that STAT3 is upregulated in glioma and plays a role in tumor progression and survival, little is known about STAT6 in this context. To obtain further evidence of STAT6 downregulation in gliomas, we performed immunohistochemical analyses using a brain glioma tissue array (see Materials and Methods). Compared to nontumor tissue, GBM tissue exhibited a significantly lower percentage of positive staining for STAT6 (Fig. 1b). Interestingly, in a primary GBM specimen in which STAT6 downregulation was not observed, STAT6 expression was clearly reduced following recurrence of glioma (Fig. 1c). STAT6 was also downregulated in astrocytoma, but not in oligodendroglioma (Fig. 1d). A transcript analysis revealed that this downregulation of STAT6 protein expression resulted from decreased mRNA production (Fig. 1e). In line with our results, a published microarray-based, high-throughput assessment (GEO DataSet record GDS1962) showed that STAT6 mRNA is reduced in glioma tissues compared with non-tumor tissue (Fig. 1f). Decreased STAT6 mRNA expression in GBM was also shown in TCGA brain in Oncomine database (Fig. 1g). Taken together, these observations demonstrate that STAT6 transcripts are downregulated in glioma tissues, leading to reduced STAT6 protein expression.

\section{DNMT1-dependent DNA methylation inhibits STAT6 expression}

To establish the mechanisms underlying downregulation of STAT6 transcript levels in human glioma, we first investigated the possibility of epigenetic silencing via $\mathrm{CpG}$ island hypermethylation. One CpG island upstream of the transcription start site of STAT6 gene $(+756$ to +1033 $\mathrm{bp})$, containing $14 \mathrm{CpG}$ sites, was predicted using MethPrimer (http://www.urogene.org/methprimer/) (Fig. 2a). An examination of methylation status in non-tumor tissue and glioma tissue using methylation-specific PCR (MSP) revealed that $\mathrm{CpG}$ islands in the STAT6 promoter were more frequently methylated in high-grade gliomas than low grade gliomas, while relatively weak methylation was found in non-tumor tissue (Fig. 2b). To validate these findings, we analyzed $14 \mathrm{CpG}$ sites located at the core of the CpG islands in non-tumor tissue and glioma tissue using bisulfite sequencing, which has increased sensitivity compared with MSP. In line with the results of MSP analyses, samples from high-grade gliomas showed prominent methylation (Fig. 2c), demonstrating a strong correlation between STAT6 downregulation and methylation of the STAT6 promoter in glioma.

Next, we explored whether promoter methylation directly mediates the silencing of STAT6 in glioma tissue. First, we examined whether STAT6 expression was increased by treatment with 5-aza-2-deoxycytidine (5-Aza), a DNMT inhibitor. In the GBM cell lines, U373MG and 
A

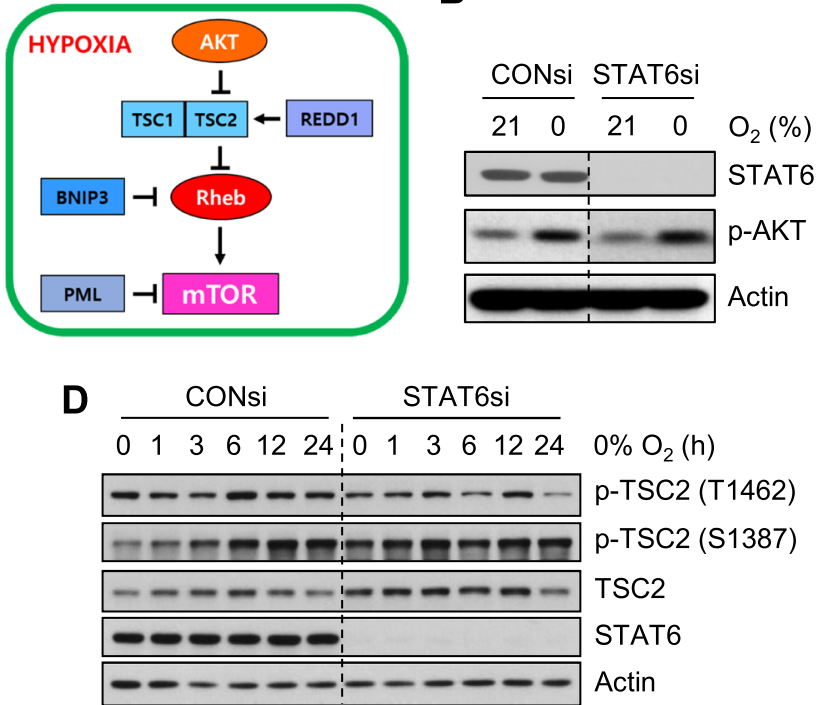

E

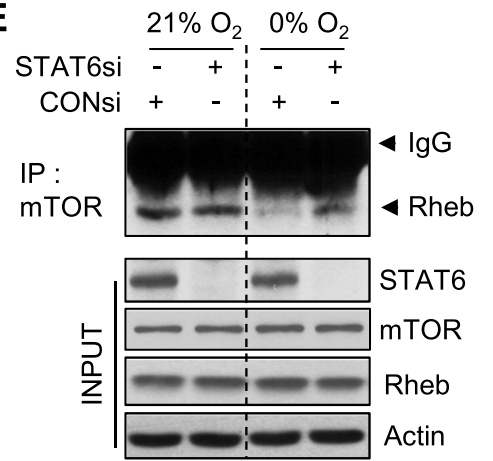

J
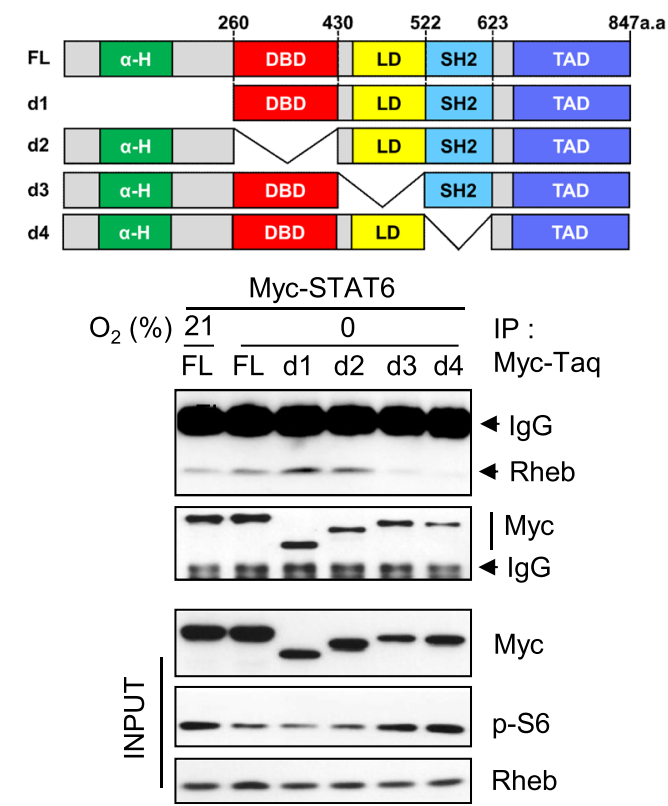

F
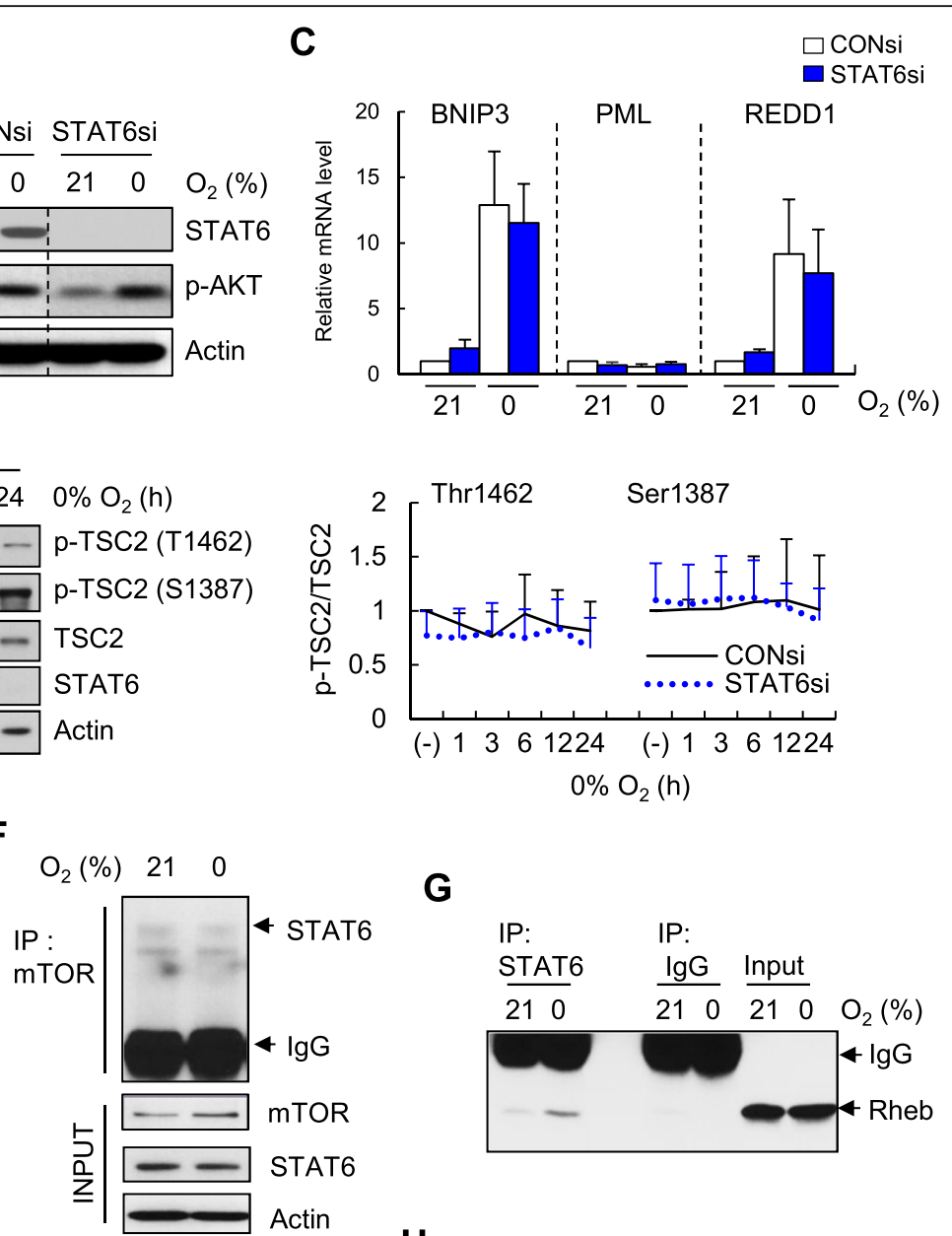

G
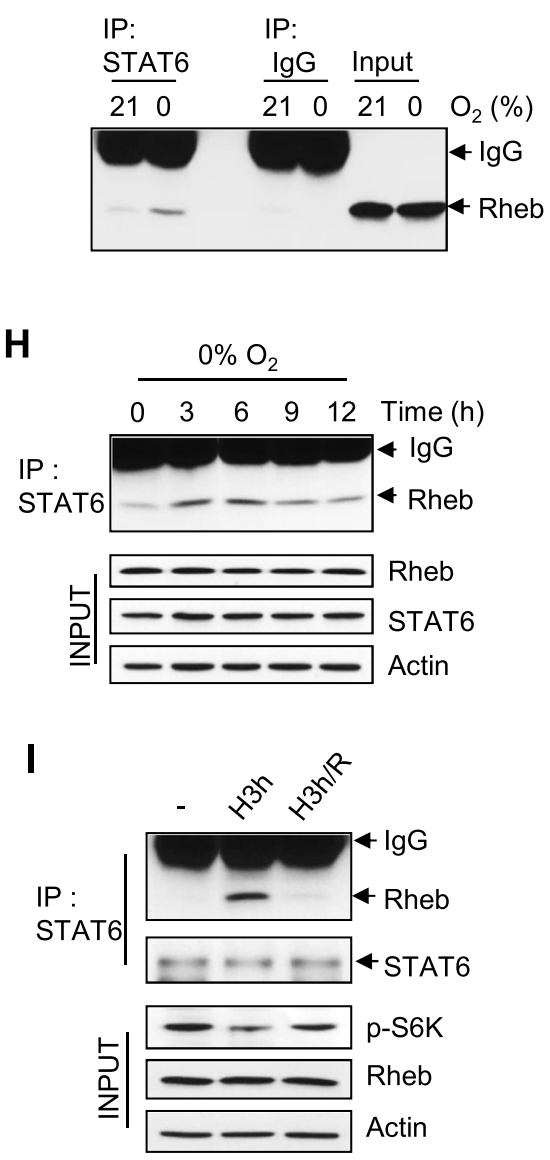

Fig. 5 (See legend on next page.) 
(See figure on previous page.)

Fig. 5 STAT6 interacts with Rheb and inhibits mTOR signaling under hypoxic conditions. a Schematic of inhibition of mTOR signaling under hypoxic conditions. b C U87MG cells transfected with non-targeting control siRNA (CONsi) or pooled siRNA targeting STAT6 (STAT6si) were exposed to $0 \% \mathrm{O}_{2}$ for $18 \mathrm{~h}$, followed by immunoblotting for STAT6 and p-AKT (b), and quantification of BNIP3, PML, and REDD1 mRNA by RTqPCR (c). d Immunoblot of p-TSC2, TSC2, and STAT6 in cells exposed to $0 \% \mathrm{O}_{2}$ for the indicated periods (left) and summary data (right) showing p-TSC2 levels, expressed as fold changes relative to that in CONsi-transfected cells exposed to $21 \% \mathrm{O}_{2}$. e Immunoblot of Rheb in mTOR immunoprecipitates (IPS) from U87MG cells transfected with CONsi or STAT6si and exposed to $21 \%$ or $0 \% \mathrm{O}_{2}$ for $10 \mathrm{~h}$. f Immunoblot of STAT6 in mTOR IPs from U87MG cells exposed to $0 \% \mathrm{O}_{2}$ for $6 \mathrm{~h} . \mathbf{g}$ Immunoblot of Rheb in STAT6 IPs from U87MG cells exposed to 0\% $\mathrm{O}_{2}$ for $10 \mathrm{~h}$. IgG IPs were used as a negative control. $\mathbf{h}$ Immunoblot of Rheb in STAT6 IPs from U87MG cells exposed to 0\% $\mathrm{O}_{2}$ for the indicated periods. $\mathbf{i}$ Immunoblot of Rheb in STAT6 IPs from U87MG cells exposed $0 \% \mathrm{O}_{2}$ for $3 \mathrm{~h}$ (H3h), followed by reoxygenation for $12 \mathrm{~h}$ (H3h/R). $\mathbf{j}$ Top, schematic of STAT6 deletion mutants. a-H, a-helicase domain; DBD, DNA-binding domain; LD, linker domain; SH2: src homology 2 domain; TAD, transactivation domain. FL, full length; d1-d4, deletion of residues 1-260 (d1), 261-430 (d2), 431-522(d3), or 523-622 (d4), respectively. Bottom, immunoblot of Rheb in Myc-tagged STAT6 (Myc-STAT6) IPs from U373MG cells transfected with the indicated Myc-STAT6 deletions and exposed to $0 \% \mathrm{O}_{2}$ for $6 \mathrm{~h}$

LN229, STAT6 expression was barely detectable and methylation was more frequent compared with U87MG cells (Fig. 2d). In U373MG cells, 5-Aza induced a concentration-dependent increase in STAT6 mRNA and protein (Fig. 2e). However, the histone deacetylase inhibitor trichostatin A (TSA), had little effect on STAT6 expression (Additional file 1: Figure S1), suggesting that methylation and not acetylation of DNA plays a dominant role in STAT6 silencing in glioma.

To further confirm the involvement of DNMT, we examined STAT6 expression level following small interfering RNA (siRNA)-mediated knockdown of the major mammalian DNMTs, DNMT1, DNMT3a and DNMT3b. In U373MG cells, STAT6 expression was restored by silencing DNMT1 with DNMT1si, but not by silencing either DNMT3a or DNMT3b (Fig. 2f). We further found that the extent to which STAT6 expression increased in U373MG cells was dependent on the concentration of DNMT1si (Fig. $2 g$ and h). Similar effects were observed in experiments using LN229 cells (Fig. 2i). Taken together, these results suggest a role for DNMT1 in STAT6 silencing in glioma.

\section{STAT6 expression regulates HIF-1a protein synthesis}

Next, we explored the functional relevance of STAT6 downregulation in GBM cells. Given the characteristic hypoxic microenvironment of glioma, we initially assessed whether STAT6 is related to HIF-1 $\alpha$ expression, since HIF- $1 \alpha$ is the critical mediator to hypoxic adaptation and survival. Experiments were performed using U87MG cells with high STAT6 expression and low methylation levels. Interestingly, while siRNA-mediated knockdown of STAT6 did not induce significant differences in HIF-1 $\alpha$ mRNA levels under hypoxia (Fig. 3a), HIF-1 $\alpha$ protein levels were clearly increased (Fig. 3b).

We then tested whether STAT6 affects HIF-1 $\alpha$ accumulation through regulation of its protein degradation. In the presence of cycloheximide ( $\mathrm{CHX}$ ) to block de novo protein synthesis, the half-life of HIF-1 $\alpha$ was similar in control and STAT6 knockdown cells (Fig. 3c), indicating that STAT6 is not involved in HIF- $1 \alpha$ protein degradation. An assay kit that detects only newly synthesized protein confirmed that de novo synthesis of HIF-1 $\alpha$ was increased in the absence of STAT6 (Fig. 3d).

To validate this finding, we performed STAT6 gain-offunction experiments in U373MG cells with barely detectable STAT6 and high promoter methylation levels. While HIF- $1 \alpha$ mRNA levels showed no differences under hypoxic conditions (Fig. 3e), HIF-1 $\alpha$ protein expression was increased in mock-transfected cells under hypoxia and conversely reduced in STAT6-overexpressing cells (Fig. 3f). This result was supported by immunofluorescence (IF) staining showing decreased HIF- $1 \alpha$ intensity in STAT6expressing cells (Fig. 3g). Assessment of protein stability using cycloheximide revealed a similar half-life of HIF- $1 \alpha$ in control and STAT6-overexpressing cells (Fig. 3h), further confirming the non-involvement of STAT6 in HIF- $1 \alpha$ protein degradation. These findings were supported using an assay kit that specifically detects newly synthesized protein (Fig. 3i).

\section{Regulation of HIF-1a protein synthesis by STAT6 is mediated via mTOR signaling}

Mammalian target of rapamycin (mTOR) regulates numerous components of protein synthesis, including ribosomal proteins and initiation and elongation factors (Fig. 4a). Since our data suggest that STAT6 inhibits HIF-1 $\alpha$ at the translational level, we assessed the involvement of mTOR signaling using rapamycin, a chemical inhibitor of mTOR. Rapamycin effectively suppressed hypoxia-induced HIF- $1 \alpha$ in both control and STAT6 knockdown cells (Fig. 4b). In view of the finding that mTOR pathways comprise S6K-S6 and 4E-BP1 (Fig. 4a), we further examined the activation status of downstream effectors of mTOR. Phosphorylation levels of both S6K and its target S6, which were decreased in a hypoxic microenvironment, were partially restored in the absence of STAT6 while the total protein levels of S6K and 56 showed no changes (Fig. 4c). In the case of 4E$\mathrm{BP} 1$, another key target of mTOR, both phosphorylated and total protein levels were increased in hypoxia, which 
A

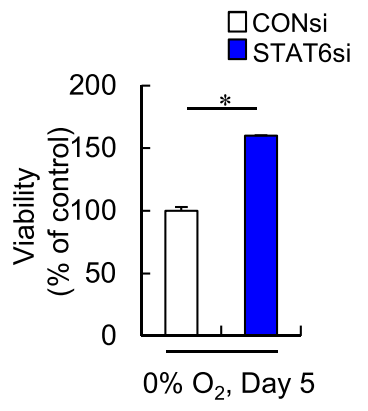

C

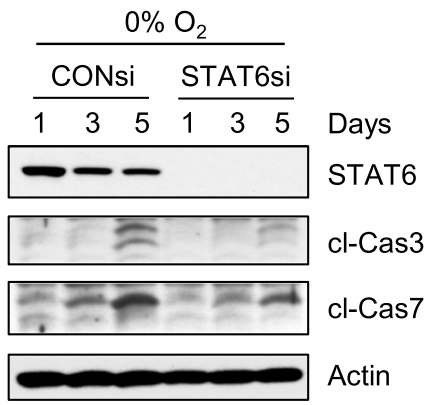

B

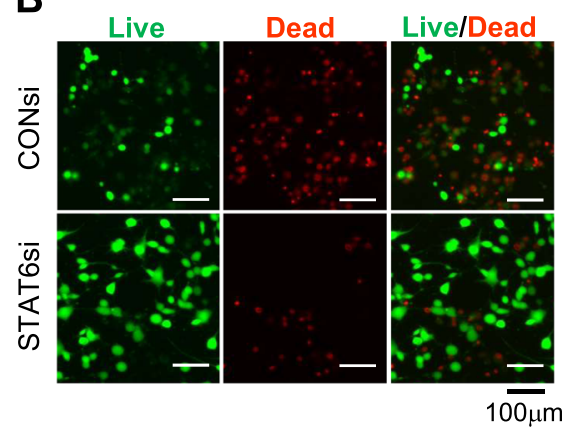

D $\quad 21 \% \mathrm{O}_{2} \quad 0 \% \mathrm{O}_{2}$

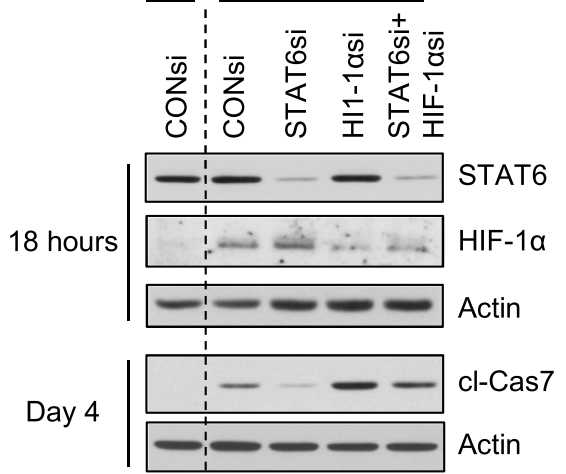

E
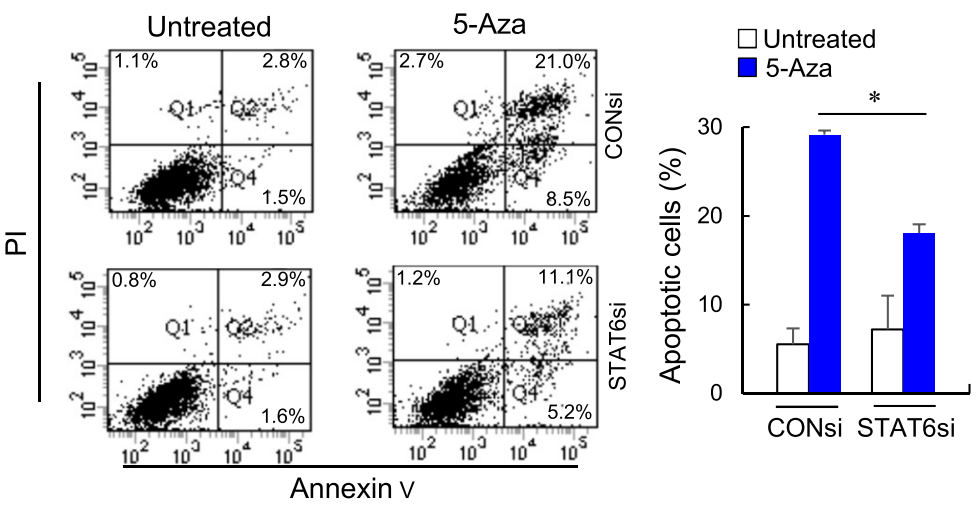

G

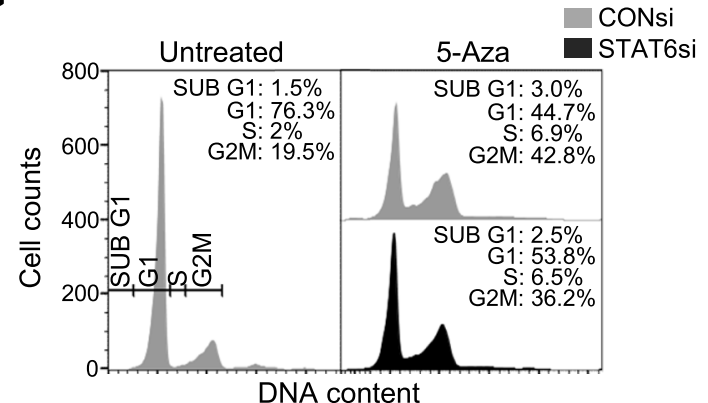

$\mathbf{F}$

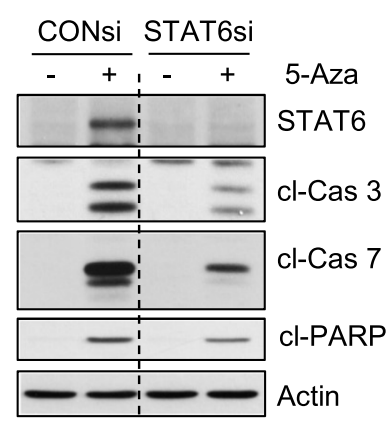

Fig. 6 (See legend on next page.)

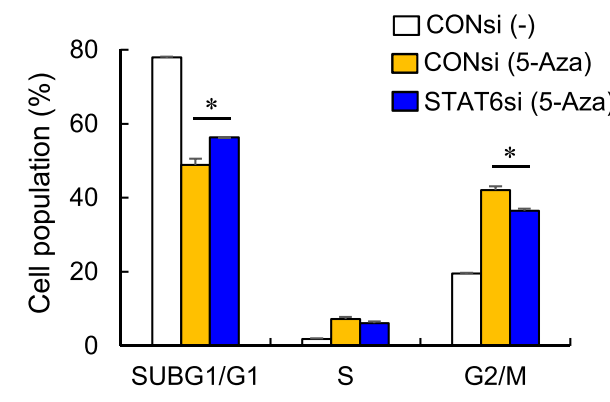


(See figure on previous page.)

Fig. 6 STAT6 regulates glioma cell viability. a, b U87MG cells transfected with CONsi or STAT6si were exposed to 0\% $\mathrm{O}_{2}$ for 5 days, and then assessed by WST viability assay (a) and LIVE (green)/DEAD (red) assay (b). Results are presented as means \pm SD (error bars) of three independent experiments ( ${ }^{*} p<0.05$ vs. CONsi-transfected cells). c Immunoblot of STAT6, cleaved (cl) caspase 3 (Cas 3), and cl-Cas 7 in siRNA-transfected U87MG cells exposed to $0 \% \mathrm{O}_{2}$ for indicated days. d Immunoblot of STAT6, HIF-1a, and cl-Cas 7 in indicated siRNA-transfected U87MG cells exposed to $0 \% \mathrm{O}_{2}$ for indicated times. e, f Two days after transfecting with CONsi or STAT6si, U373MG cells were incubated with or without 5Aza (300 nM) for 2 days, and incubated in fresh medium (without drugs) for 6 days. Flow cytometry dot plots of annexin $V$ and propidium iodide (PI) staining and summary data showing the percentage of annexin V-positive cells (e) and immunoblot of STAT6, cl-Cas-3 and -7, and cl- PARP (f). Results are presented as means \pm SD (error bars) of three independent experiments ( ${ }^{*} p<0.05$ ). $\mathbf{g}$ Flow cytometry histogram plots of PI-stained DNA content (left) and summary data (right) showing the percentage of cells in each cell cycle phase. Results are presented as means \pm SD (error bars) of three independent experiments $\left({ }^{*} p<0.05\right)$

were suppressed in the absence of STAT6 (Fig. 4d). The corresponding increase in 4E-BP1 mRNA expression in hypoxia and its suppression by STAT6si (Fig. 4e) indicated that phosphorylation and protein levels reflect transcriptional patterns. In STAT6 knockdown cells, restoration of the suppressed mTOR signaling in hypoxia facilitated eIF4E binding to eIF4G (Fig. 4f).

In line with STAT6 knockdown experiments, hypoxiainduced suppression of p-S6K-S6K signaling was further decreased in STAT6-overexpressing U373MG cells (Additional file 1: Figure S2A). However, protein and transcript levels of 4E-BP1 and p-4E-BP1 displayed no significant changes following STAT6 overexpression (Additional file 1: Figure S2B and C).

\section{STAT6 interacts directly with Rheb, leading to mTOR inhibition}

The mechanism underlying STAT6-mediated inhibition of mTOR activity under hypoxia was further investigated. The TSC2-Rheb signaling axis induced suppression of mTOR activity under hypoxia (Fig. 5a). However, STAT6 did not affect activity of the upstream kinase AKT (Fig. 5b). No significant differences in mRNA induction of genes regulating DNA damage and development, including REDD 1, BNIP3 and PML (Fig. 5c), or TSC2 phosphorylation at T1462 or S138 (Fig. 5d) were evident between control-, and STAT6 siRNA-transfected U87MG in hypoxic conditions.

Accordingly, we considered the possibility that STAT6 interferes with Rheb-mTOR interactions, thereby suppressing mTOR activity. Interestingly, hypoxia-induced RhebmTOR dissociation was maintained in STAT6 knockdown cells (Fig. 5e). To further examine the effects of STAT6 on Rheb-mTOR binding, we analyzed the interactions of STAT6 with Rheb or mTOR. STAT6 interacted with Rheb (Fig. 5 g) but not mTOR (Fig. 5f), with a peak representing optimal binding at 3-6 h after hypoxia (Fig. 5h). Moreover, under reoxygenation conditions, hypoxia-induced STAT6 binding to Rheb was suppressed (Fig. 5i), indicating the necessity of hypoxic conditions for STAT6-Rheb interactions. For further analysis of STAT6-Rheb interactions, we mapped the Rheb-binding region of STAT6 using deletion mutants of each domain. Deletion of either the Linker
Domain (LD) or SH2 domain (SH) abolished STAT6 binding to Rheb under hypoxic conditions (Fig. 5j). Upon inhibition of STAT6-Rheb interactions (via transfection of $\mathrm{d} 3$ or d4), S6 phosphorylation was restored, even in hypoxic conditions (Fig. 5j, INPUT), signifying inhibitory effects of STAT6 on mTOR signaling through interactions with Rheb.

\section{STAT6 expression promotes glioma cell death}

To address the functional significance of STAT6-regulated HIF- $1 \alpha$ expression in GBM cells, we initially examined the impact of STAT6 on cell viability under hypoxic microenvironments. In U87MG cells, siRNA-mediated knockdown of STAT6 led to increased cell viability (Fig. 6a and b) and decreased expression of apoptosis markers (Fig. 6c). Next, we examined the interrelationships among STAT6, HIF-1 $\alpha$ and apoptosis by examining the expression patterns of HIF- $1 \alpha$ and apoptosis markers at $18 \mathrm{~h}$ and 4 days after hypoxia, respectively. Hypoxia-induced cell death was decreased in the presence of STAT6si, which was reversed in double knockdown cells. In parallel, hypoxia-induced cell death was potentiated by HIF-1 $1 \alpha$ si and, conversely, attenuated in double knockdown cells, indicating that HIF-1 $\alpha$ expression is dependent on STAT6 (Fig. 6d).

In STAT6 gain-of function experiments, STAT6overexpressing U373MG cells exhibited decreased viability (Additional file 1: Figure S3A) along with increased expression of apoptosis markers (Additional file 1: Figure S3B and C).

In recent years, hypomethylating agents, such as 5azacitidine and decitabine (5-Aza), have been clinically applied for treatment of myelodysplasia. Interestingly, these agents exert DNA methyltransferase (DNMT) inhibition effects at significantly lower concentrations than those required for cancer cell killing effects. Moreover, effects are sustained even after washout of drugs. In our cell culture model, the apoptotic cell content was significantly induced 3 days after washout of 5-Aza at concentrations $\geq 100 \mathrm{nM}$, which continued to increase until 6 days (Additional file 1: Figure S4).

To test whether epigenetic restoration of STAT6 mediates apoptotic effects of 5-Aza, STAT6-silenced U373MG cells were treated with 5-Aza. Transient 5-Aza treatment increased apoptotic cell death to $29 \pm 0.5 \%$, which was 


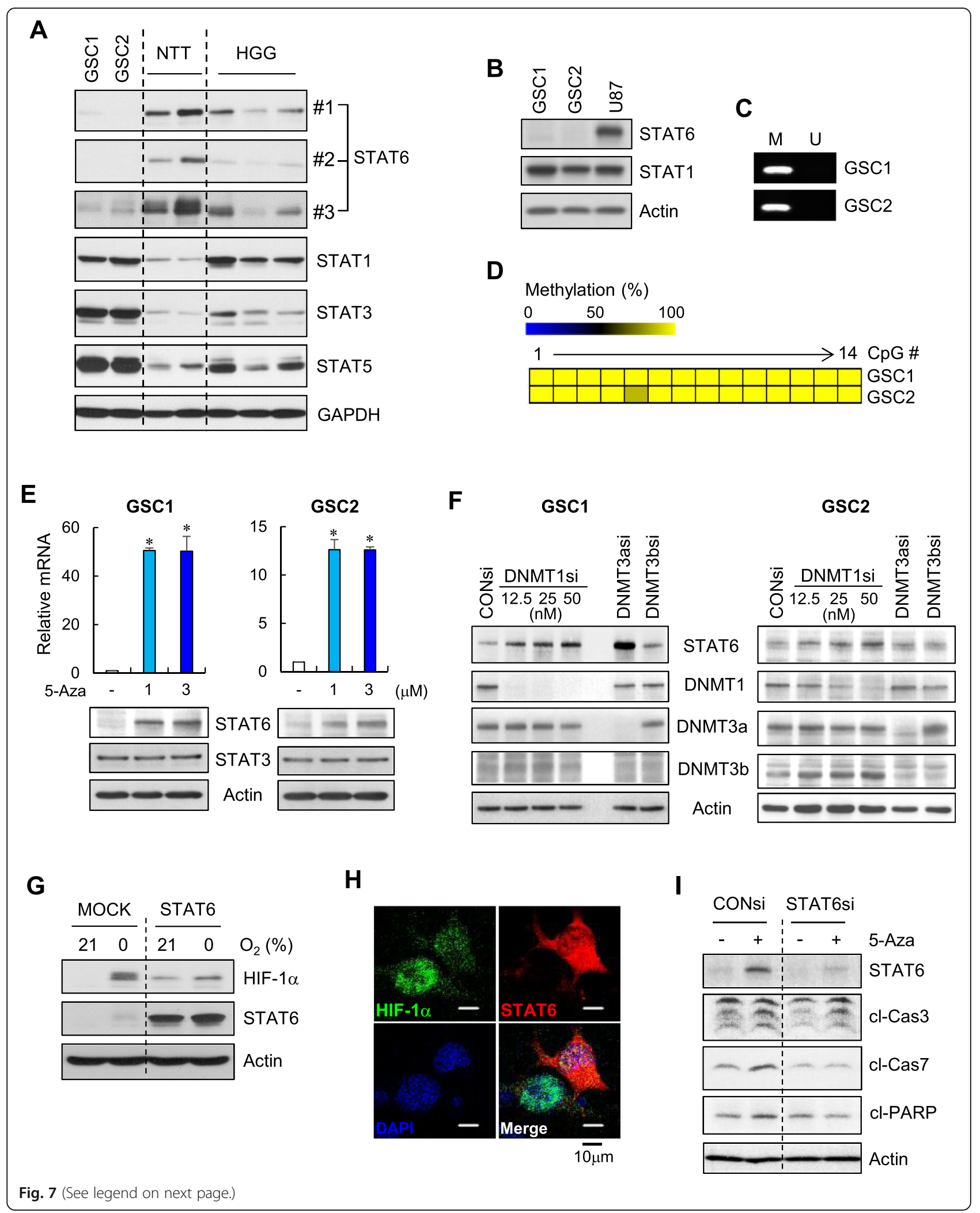


(See figure on previous page.)

Fig. 7 STAT6 expression is downregulated in GSCs. a Immunoblot of the indicated STATs in patient-derived GSCs (GSC1 and GSC2), non-tumor brain tissue (NTT), and high-grade glioma (HGG) tissue. STAT6 antibodies: \#1, Cell Signaling Technology; \#2, BD Biosciences; \#3, Santa Cruz. b-d Immunoblot of STAT6 and STAT1 (b) and MSP (c) and bisulfite sequencing (d) of STAT6 promoter CpG islands in GSCs. e GSCs were treated with the indicated concentrations of 5-Aza for $72 \mathrm{~h}$, followed by quantification of STAT6 mRNA by RT-qPCR (top) and immunoblotting for STAT6 and STAT3 (bottom). Results are presented as means \pm SD (error bars) of three independent experiments $\left({ }^{*} p<0.05\right)$. $\mathbf{f}$ Immunoblot of STAT6 and the indicated DNMTs in GSCs transfected with the indicated DNMT siRNA or control siRNA. $\mathbf{g}, \mathbf{h}$ Immunoblot of HIF-1a and STAT6 in GSC1 cells transfected with empty vector (MOCK) or Myc-tagged full-length STAT6 (STAT6) and exposed to 21\% or 0\% $\mathrm{O}_{2}$ for $18 \mathrm{~h}$ (g) and IF staining of HIF1a in STAT6-transfected GSC1 cells exposed to $0 \% \mathrm{O}_{2}$ for $18 \mathrm{~h} \mathrm{(h).} \mathrm{i} \mathrm{CONsi-} \mathrm{or} \mathrm{STAT6si-transfected} \mathrm{GSC1} \mathrm{cells} \mathrm{were} \mathrm{treated} \mathrm{as} \mathrm{in} \mathrm{Fig.} \mathrm{6e,} \mathrm{followed}$ by immunoblotting for indicated proteins

decreased to $18 \pm 2.5 \%$ in the presence of STAT6si (Fig. 6e). Effects of STAT6 knockdown on apoptosis were further confirmed by reduced cleavage of capase-3, caspase- 7 and poly-(ADP-ribose) polymerase (PARP) (Fig. 6f). Additionally, 5-Aza induced arrest of cells in the G2/M phase under the same conditions as in Fig. 6e, which was significantly reduced upon STAT6 knockdown (Fig. 6g). Collectively, these results suggest that the effects of transient, low-dose 5-Aza on cell death and the cell cycle are primarily, but not exclusively, dependent on STAT6 re-expression in glioma cells.

In addition to its cell-killing effects, transient exposure to low doses of 5-Aza is reported to exert sustained antitumor effects through induction of immune signaling [18, 47]. We further examined whether epigenetic re-expression of STAT6 by 5 -Aza is the mechanism mediating expression of immune-related genes. Indeed, several immune-related genes, including interleukin (IL)-1 $\beta, \mathrm{C}$-C motif chemokine ligand (CCL)2, CCL20, IL-8, ICAM1 intercellular adhesion molecule 1 (ICAM1), nuclear factor of activated T cells 2 (NFATC2) and Janus kinase 2 (JAK2), that were significantly induced by low doses of 5-Aza were suppressed upon STAT6 knockdown (Additional file 1: Figure S5), clearly demonstrating dependence of expression on STAT6. Our results support the utility of 5-Aza as an effective chemotherapeutic agent with immune modulating capacity as well as cell-killing effects.

\section{STAT6 expression is downregulated in glioma stem-like cells}

Glioma stem-like cells (GSCs) have been implicated in glioma initiation, progression, therapeutic resistance and tumor recurrence, and are thus critical targets for therapy [22]. Therefore, we determined whether our findings could be extended to GSCs. To this end, we established and characterized two patient-derived GSC cell lines, GSC1 and GSC2 (Additional file 1: Figure S6). An examination of STAT protein expression levels showed that STAT6 levels were significantly lower in GSCs compared with nontumor tissue, whereas levels of STAT1, STAT3 and STAT5 were significantly higher (Fig. 7a). Notably, STAT6 levels in GSCs were even lower than those in high-grade glioma tissues (Fig. 7a). Next, we examined STAT6 promoter methylation status in GSCs. MSP and bisulfite sequencing showed that the STAT6 promoter was highly methylated in GSCs (Fig. 7b). Furthermore, 5-Aza treatment and DNMT1 knockdown successfully restored STAT6 expression in GSCs (Fig. 7c and d), demonstrating promotermethylation-dependent STAT6 silencing in GSCs. Intriguingly, although STAT6 silencing was only dependent on DNMT1 in GBM cell lines (Fig. 2), in case of GSC1, both DNMT3a and DNMT1 contributed to STAT6 silencing (Fig. 7d). Finally, we examined the role of STAT6 in HIF$1 \alpha$ accumulation and 5-Aza effects on GSCs. STAT6 overexpression significantly reduced HIF- $1 \alpha$ levels in hypoxic GSCs (Fig. 7e and f), indicating that STAT6 downregulation may facilitate GSC survival under hypoxic conditions. In addition, 5-Aza-induced apoptosis were significantly attenuated by STAT6 knockdown.

\section{Discussion}

STATs are dysregulated in a broad range of cancer types. In this study, we demonstrated that STAT6 is downregulated in human glioma specimens as a result of $\mathrm{CpG}$ methylation of the STAT6 promoter. STAT6 downregulation, in turn, promotes HIF-1 $\alpha$ protein synthesis under hypoxic conditions through activation of the mTOR signaling pathway, leading to enhanced cell survival. Suppression of mTOR-S6K-S6 by STAT6 is achieved through direct interactions with Rheb. Our experiments indicate that STAT6 restoration using hypomethylating agents, such as 5-azacitidine and decitabine, can promote gliomal cell death in STAT6-silenced GBM. The results obtained in this study extend the potential clinical application of hypomethylating agents for solid tumors, including GBM, requiring multi-modal treatment and precision medicine.

Accumulating evidence indicates that aberrant DNA methylation contributes to GBM pathology [11, 27]. In particular, methylation-specific genomic analyses have identified hypermethylation of notable genes in GBM, including MGMT [49]. Our expression profiling similarly demonstrated frequent downregulation of STAT6 in human GBM specimens as a result of STAT6 promoter CpG methylation, which is maintained by DNMTs. In our experiments, STAT6 was downregulated under hypoxia in GBM cells, which was reversed by 5 -Aza (Additional file 1: Figure S7A and B). Moreover, reoxygenation conditions 
rescued STAT6 expression (Additional file 1: Figure S7C) and hypoxia-induced promoter methylation was evident with bisulfite sequencing (Additional file 1: Figure S7D). Consistent with our findings, several reports have shown that hypoxia downregulates a number of tumor suppressor genes, including mutL homolog 1 (MLH1), Runt-related transcription factor 3 (RUNX3) and von Hippel-Lindau tumor suppressor (pVHL), through epigenetic mechanisms $[19,23,29]$, supporting a potential tumor suppressor function of STAT6.

Hypoxia is a characteristic feature of GBM [16]. Given that HIF- $1 \alpha$ is a major regulator in hypoxic environments, our finding that STAT6 negatively regulates HIF-1 $\alpha$ suggests an important role in hypoxic GBM. Data from this study showed that STAT6 silencing promotes HIF- $1 \alpha$ expression at the translational level via stimulating mTOR-S6K-S6 activity. In keeping with these results, loss of specific tumor suppressors, such as promyelocytic leukemia protein (PML) or tuberous sclerosis proteins 1 and 2 (TSC1 and TSC2), has been shown to promote HIF-1 $\alpha$ expression through suppression of hypoxia-induced inhibition of mTOR [2, 4]. Mechanistically, STAT6 suppresses mTOR and downstream S6K and $\mathrm{S} 6$ phosphorylation through direct interactions with Rheb. Our data revealed a non-transcriptional function of STAT6, leading to mTOR inhibition. In line with our results, there are several reports regarding non-transcriptional function of STATs. For example, STAT3 is reported to involve in microtubule organization, mitochondrial bioenergetic function, and chromatin regulation $[32,46,48]$.

Apart from its inhibitory effects on mTOR signaling, hypoxia promoted transcript and protein expression of 4E-BP1, which was suppressed by STAT6si (Fig. 4d and e). Since 4E-BP1 itself is increased under hypoxia conditions, we were unsure whether the increase in $\mathrm{p}-4 \mathrm{E}-\mathrm{BP} 1$ simply reflects an increase in non-functional transcripts but both were suppressed by STAT6si. However, eIF4E phosphorylation and its interactions with eIF4G were significantly enhanced in hypoxic STAT6 knockdown cells (Fig. 4f). Although mTOR is considered a promising target for cancer therapy, targeting mTOR using rapamycin has produced limited beneficial effects in patients [24]. One proposed reason for this unsatisfactory outcome is incomplete targeting of 4E-BP1 phosphorylation [8]. In addition, 4E-BP1 silencing renders protein synthesis resistant to mTOR inhibitors [28]. In this respect, our finding that STAT6 is involved in regulating 4E-BP1 expression could be exploited for rapamycin therapy. Specifically, if 4E-BP1 expression could be regulated by STAT6, STAT6 restoration and the resulting increase in 4E-BP1 could overcome rapamycin-resistant 4E-BP1 phosphorylation in STAT6-silenced GBM. Accordingly, drugs that restore STAT6 expression, such as 5-Aza, may be useful for cancer therapy in combination with rapamycin.
Individual STAT family members not only have discrete functions, they also sometimes play redundant and overlapping roles. For example, constitutively activated STAT3 antagonizes the proapoptotic effects of activated STAT1 in fibroblasts [42]. However, in the absence of STAT1, STAT3 activation by IFN $-\gamma$ is potentiated and prolonged [38], whereas in MEFs lacking STAT3, IL-6 mediates an IFN- $\gamma$-like response, including prolonged activation of STAT1 [9]. Similar to the case of STAT1 and STAT3, it is likely that STAT3 and STAT6 reciprocally regulate each other in GBM. We observed frequent STAT6 downregulation in conjunction with constitutive STAT3 activation in GBM samples tested (Fig. 1a). Further, in malignant glioma cells, it has shown that IL-4 induces aberrant STAT3 activation, but not STAT6 activation [39], suggesting that a defective STAT6 signal might drive STAT3 activation. Although more studies are needed to elucidate the specific relationship between STAT3 and STAT6 in GBM, our data collectively suggest that STAT6 downregulation shifts the balance in favor of GBM pathology.

In addition to its prominent role in adaptive immunity, STAT6 is a critical mediator of viral defense signaling [5]. One significant finding of this study is that STAT6 mediates the induction of chemokines (CCL2, CCL20 and IL8 ) by 5 -Aza (Additional file 1: Figure S5). Innate immune signaling pathways in host immune and tumor cells have emerged as determinants of responses and resistance to immunotherapy [50]. The activation of tumor-intrinsic innate immune signaling pathways could induce attraction of lymphocytes to tumors, thereby enhancing immunotherapies [31]. Indeed, a recent report has shown that 5Aza is capable of activating this pathway and inducing sensitization to immune checkpoint blockade [7]. Given that various immunotherapies are being actively studied for treatment of advanced glioma [35], it may be possible that 5-Aza can sensitize GBM cancer cells to immunotherapy, and that STAT6, a critical mediator of chemokine induction, could be a key player in this sensitization mechanism.

GSCs play a central role in cancer initiation and therapeutic resistance, and thus have become a target of GBM therapy [22]. In this context, we demonstrated STAT6 downregulation, its negative regulation of HIF- $1 \alpha$, and its mediation of 5-Aza effects in patient-derived GSCs (Fig. 7). Because HIF- $1 \alpha$ is critical for GSC maintenance [37, 43], STAT6 downregulation may favor HIF-1 $\alpha$ accumulation in GSCs under hypoxic conditions, thereby contributing to GSC maintenance and GBM progression. Furthermore, 5Aza is capable of targeting GSCs [40]; thus, restoration of STAT6 may be another mechanism underlying the actions of 5-Aza against GSCs.

Despite recent advances in cancer therapy, the efficacy of anticancer therapeutics remains limited, possibly owing to the complex heterogeneity of cancer cells; thus, some 
patients still need novel therapeutic agents. The same is true of GBM treatment. Although validation in in vivo, followed by clinical trial is needed, our results suggest that restoration of STAT6 is one such novel therapy. Namely, in GBM in which STAT6 is downregulated, restoring STAT6 would suppress HIF-1 $\alpha$ protein synthesis and induce STAT6-regulated immune responses and apoptosis, thereby leading to GMB suppression. Taken together, our data reveal novel roles and mechanisms of action of STAT6 in GBM and propose STAT6 restoration as a promising strategy for GBM therapy.

\section{Conclusions}

Here we show that STAT6 is epigenetically silenced in some cases of malignant glioma, ultimately preventing cancer cell death by inducing HIF-1 $\alpha$ expression via the mTOR-S6K-S6 pathway. mTOR activation upon STAT6 knockdown occurs as a result of suppression of direct interactions between STAT6 and Rheb that inhibit HIF$1 \alpha$ translation. Data from our study support a previously uncharacterized role of STAT6 in glioma cell survival and suggest that epigenetic restoration of STAT6 with the aid of DNA methyltransferase inhibitors, including 5-aza-2'-deoxycytidine (decitabine), could be applied as therapy for STAT6-silenced glioma.

\section{Additional files}

Additional file 1: Figure S1. Histone acetylation does not contribute to STAT6 silencing. Figure S2. STAT6 overexpression suppresses the mTORS6K-S6 pathway in U373MG cells during hypoxia. Figure S3. STAT6overexpressing U373MG cells exhibited increased apoptosis during hypoxia. Figure S4. Experimental validation for low dose, transient 5-Aza treatment. Figure S5. 5-Aza-induced STAT6 expression increases the expression of immune-related genes. Figure S6. Characterization of GSCs. Figure S7. STAT6 expression is epigenetically silenced during hypoxia. (PDF $990 \mathrm{~kb}$ )

Additional file 2: Table S1. Detailed information on glioma samples used in this study. Table S2. The primers used for qRT-PCR. Table S3. Targets CpG islands and the primers for pyrosequencing. Table S4. PCR Primers for Expressing Vectors. (PDF $413 \mathrm{~kb}$ )

\begin{abstract}
Authors' contributions
SJP performed the biological experiments, analyzed the data. HK provided technical support. SHK provided human specimens. E-hJ provided advice on the overall study. SJP and IJ designed the study and wrote the manuscript. IJ led the overall study. All authors read and approved the final manuscript.
\end{abstract}

\section{Funding}

This work was supported by the National Research Foundation of Korea (NRF) grant funded by the Korea government (MSIT) (NRF-2012R1A5A2048183).

\section{Availability of data and materials}

The datasets used and/or analyzed during the present study are available from the corresponding author on response request.

\section{Competing interests}

The authors declare that they have no competing interests.

\section{Author details}

'Department of Pharmacology, School of Medicine, Ajou University, Suwon 16499, South Korea. ${ }^{2}$ Chronic Inflammatory Disease Research Center, Ajou University, Suwon 16499, South Korea. ${ }^{3}$ Department of Neurosurgery, School of Medicine, Ajou University, Suwon 16499, South Korea.

Received: 14 July 2019 Accepted: 26 August 2019

Published online: 17 September 2019

\section{References}

1. Ahuja N, Sharma AR, Baylin SB (2016) Epigenetic therapeutics: a new weapon in the war against Cancer. Annu Rev Med 67:73-89. https://doi. org/10.1146/annurev-med-111314-035900

2. Bernardi R, Guernah I, Jin D, Grisendi S, Alimonti A, Teruya-Feldstein J, Cordon-Cardo C, Simon MC, Rafii S, Pandolfi PP (2006) PML inhibits HIF-1alpha translation and neoangiogenesis through repression of mTOR. Nature 442:779-785. https://doi.org/10.1038/nature05029

3. Brantley EC, Benveniste EN (2008) Signal transducer and activator of transcription-3: a molecular hub for signaling pathways in gliomas. Mol Cancer Res 6:675-684. https://doi.org/10.1158/1541-7786.MCR-07-2180

4. Brugarolas J, Lei K, Hurley RL, Manning BD, Reiling JH, Hafen E, Witters LA, Ellisen LW, Kaelin WG Jr (2004) Regulation of mTOR function in response to hypoxia by REDD1 and the TSC1/TSC2 tumor suppressor complex. Genes Dev 18:2893-2904. https://doi.org/10.1101/gad.1256804

5. Chen H, Sun H, You F, Sun W, Zhou X, Chen L, Yang J, Wang Y, Tang H, Guan $Y$ et al (2011) Activation of STAT6 by STING is critical for antiviral innate immunity. Cell 147:436-446. https://doi.org/10.1016/j.cell.2011.09.022

6. Chen L, Feng P, Li S, Long D, Cheng J, Lu Y, Zhou D (2009) Effect of hypoxia-inducible factor-1alpha silencing on the sensitivity of human brain glioma cells to doxorubicin and etoposide. Neurochem Res 34:984-990. https://doi.org/10.1007/s11064-008-9864-9

7. Chiappinelli KB, Strissel PL, Desrichard A, Li H, Henke C, Akman B, Hein A, Rote NS, Cope LM, Snyder A et al (2015) Inhibiting DNA methylation causes an interferon response in Cancer via dsRNA including endogenous retroviruses. Cell 162:974-986. https://doi.org/10.1016/j.cell.2015.07.011

8. Choo AY, Yoon SO, Kim SG, Roux PP, Blenis J (2008) Rapamycin differentially inhibits S6Ks and 4E-BP1 to mediate cell-type-specific repression of mRNA translation. Proc Natl Acad Sci U S A 105:17414-17419. https://doi.org/10.1 073/pnas.0809136105

9. Costa-Pereira AP, Tininini S, Strobl B, Alonzi T, Schlaak JF, Is'harc H, Gesualdo I, Newman SJ, Kerr IM, Poli V (2002) Mutational switch of an IL-6 response to an interferon-gamma-like response. Proc Natl Acad Sci U S A 99:8043-8047. https://doi.org/10.1073/pnas.122236099

10. Dodd KM, Yang J, Shen MH, Sampson JR, Tee AR (2015) mTORC1 drives HIF-1alpha and VEGF-A signalling via multiple mechanisms involving 4E-BP1, S6K1 and STAT3. Oncogene 34:2239-2250. https:/doi.org/10.1038/onc.2014.164

11. Etcheverry A, Aubry M, de Tayrac M, Vauleon E, Boniface R, Guenot F, Saikali S, Hamlat A, Riffaud L, Menei P et al (2010) DNA methylation in glioblastoma: impact on gene expression and clinical outcome. BMC Genomics 11:701. https://doi.org/10.1186/1471-2164-11-701

12. Fujiwara S, Nakagawa K, Harada H, Nagato S, Furukawa K, Teraoka M, Seno T, Oka K, Iwata S, Ohnishi T (2007) Silencing hypoxia-inducible factor-1alpha inhibits cell migration and invasion under hypoxic environment in malignant gliomas. Int J Oncol 30:793-802

13. Harris AL (2002) Hypoxia--a key regulatory factor in tumour growth. Nat Rev Cancer 2:38-47. https://doi.org/10.1038/nrc704

14. Heddleston JM, Li Z, McLendon RE, Hjelmeland AB, Rich JN (2009) The hypoxic microenvironment maintains glioblastoma stem cells and promotes reprogramming towards a cancer stem cell phenotype. Cell Cycle 8:32743284. https://doi.org/10.4161/cc.8.20.9701

15. Jaenisch R, Bird A (2003) Epigenetic regulation of gene expression: how the genome integrates intrinsic and environmental signals. Nat Genet 33(Suppl): 245-254. https://doi.org/10.1038/ng1089

16. Jensen RL (2009) Brain tumor hypoxia: tumorigenesis, angiogenesis, imaging, pseudoprogression, and as a therapeutic target. J Neuro-Oncol 92: 317-335. https://doi.org/10.1007/s1 1060-009-9827-2

17. Jones PA (2012) Functions of DNA methylation: islands, start sites, gene bodies and beyond. Nat Rev Genet 13:484-492. https://doi.org/10.1038/nrg3230

18. Juergens RA, Wrangle J, Vendetti FP, Murphy SC, Zhao M, Coleman B, Sebree R, Rodgers K, Hooker CM, Franco N et al (2011) Combination epigenetic therapy has efficacy in patients with refractory advanced 
non-small cell lung cancer. Cancer Discov 1:598-607. https://doi.org/10.115 8/2159-8290.CD-11-0214

19. Kim MS, Kwon HJ, Lee YM, Baek JH, Jang JE, Lee SW, Moon EJ, Kim HS, Lee SK, Chung HY et al (2001) Histone deacetylases induce angiogenesis by negative regulation of tumor suppressor genes. Nat Med 7:437-443. https:// doi.org/10.1038/86507

20. Kontro M, Kuusanmaki H, Eldfors S, Burmeister T, Andersson El, Bruserud O, Brummendorf TH, Edgren H, Gjertsen BT, Itala-Remes M et al (2014) Novel activating STAT5B mutations as putative drivers of T-cell acute lymphoblastic leukemia. Leukemia 28:1738-1742. https://doi.org/10.1038/ leu.2014.89

21. Koskela HL, Eldfors S, Ellonen P, van Adrichem AJ, Kuusanmaki H, Andersson El, Lagstrom S, Clemente MJ, Olson T, Jalkanen SE et al (2012) Somatic STAT3 mutations in large granular lymphocytic leukemia. N Engl J Med 366: 1905-1913. https://doi.org/10.1056/NEJMoa1114885

22. Lathia JD, Mack SC, Mulkearns-Hubert EE, Valentim CL, Rich JN (2015) Cancer stem cells in glioblastoma. Genes Dev 29:1203-1217. https://doi. org/10.1101/gad.261982.115

23. Lee SH, Kim J, Kim WH, Lee YM (2009) Hypoxic silencing of tumor suppressor RUNX3 by histone modification in gastric cancer cells. Oncogene 28:184-194. https://doi.org/10.1038/onc.2008.377

24. Li J, Kim SG, Blenis J (2014) Rapamycin: one drug, many effects. Cell Metab 19:373-379. https://doi.org/10.1016/..cmet.2014.01.001

25. Li Z, Bao S, Wu Q, Wang H, Eyler C, Sathornsumetee S, Shi Q, Cao Y, Lathia J, RE ML et al (2009) Hypoxia-inducible factors regulate tumorigenic capacity of glioma stem cells. Cancer Cell 15:501-513. https://doi.org/10.1 016/j.ccr.2009.03.018

26. Liang QC, Xiong H, Zhao ZW, Jia D, Li WX, Qin HZ, Deng JP, Gao L, Zhang H, Gao GD (2009) Inhibition of transcription factor STAT5b suppresses proliferation, induces $\mathrm{G} 1$ cell cycle arrest and reduces tumor cell invasion in human glioblastoma multiforme cells. Cancer Lett 273:164-171. https://doi. org/10.1016/j.canlet.2008.08.011

27. Mack SC, Hubert CG, Miller TE, Taylor MD, Rich JN (2016) An epigenetic gateway to brain tumor cell identity. Nat Neurosci 19:10-19. https://doi. org/10.1038/nn.4190

28. Martineau Y, Azar R, Muller D, Lasfargues C, El Khawand S, Anesia R, Pelletier J, Bousquet C, Pyronnet S (2014) Pancreatic tumours escape from translational control through 4E-BP1 loss. Oncogene 33:1367-1374. https:// doi.org/10.1038/onc.2013.100

29. Mihaylova VT, Bindra RS, Yuan J, Campisi D, Narayanan L, Jensen R, Giordano F, Johnson RS, Rockwell S, Glazer PM (2003) Decreased expression of the DNA mismatch repair gene Mlh1 under hypoxic stress in mammalian cells. Mol Cell Biol 23:3265-3273

30. Miklossy G, Hilliard TS, Turkson J (2013) Therapeutic modulators of STAT signalling for human diseases. Nat Rev Drug Discov 12:611-629. https://doi. org/10.1038/nrd4088

31. Minn AJ, Wherry EJ (2016) Combination Cancer therapies with immune checkpoint blockade: convergence on interferon signaling. Cell 165:272-275. https://doi.org/10.1016/i.cell.2016.03.031

32. Ng DC, Lin BH, Lim CP, Huang G, Zhang T, Poli V, Cao X (2006) Stat3 regulates microtubules by antagonizing the depolymerization activity of stathmin. J Cell Biol 172:245-257. https://doi.org/10.1083/jcb.200503021

33. Nicolae A, Xi L, Pittaluga S, Abdullaev Z, Pack SD, Chen J, Waldmann TA, Jaffe ES, Raffeld M (2014) Frequent STAT5B mutations in gammadelta hepatosplenic T-cell lymphomas. Leukemia 28:2244-2248. https://doi.org/1 $0.1038 /$ leu.2014.200

34. Noushmehr H, Weisenberger DJ, Diefes K, Phillips HS, Pujara K, Berman BP, Pan F, Pelloski CE, Sulman EP, Bhat KP et al (2010) Identification of a CpG island methylator phenotype that defines a distinct subgroup of glioma. Cancer Cell 17:510-522. https://doi.org/10.1016/j.ccr.2010.03.017

35. Patel MA, Pardoll DM (2015) Concepts of immunotherapy for glioma. Neuro-Oncol 123:323-330. https://doi.org/10.1007/s11060-015-1810-5

36. Portela A, Esteller M (2010) Epigenetic modifications and human disease. Nat Biotechnol 28:1057-1068. https://doi.org/10.1038/nbt.1685

37. Qiang L, Wu T, Zhang HW, Lu N, Hu R, Wang YJ, Zhao L, Chen FH, Wang XT, You QD et al (2012) HIF-1alpha is critical for hypoxia-mediated maintenance of glioblastoma stem cells by activating notch signaling pathway. Cell Death Differ 19:284-294. https://doi.org/10.1038/cdd.2011.95

38. Qing Y, Stark GR (2004) Alternative activation of STAT1 and STAT3 in response to interferon-gamma. J Biol Chem 279:41679-41685. https://doi. org/10.1074/jbc.M406413200
39. Rahaman SO, Vogelbaum MA, Haque SJ (2005) Aberrant Stat3 signaling by interleukin-4 in malignant glioma cells: involvement of IL-13Ralpha2. Cancer Res 65:2956-2963. https://doi.org/10.1158/0008-5472.CAN-04-3592

40. Riccadonna C, Yacoub Maroun C, Vuillefroy de Silly R, Boehler M, Calvo Tardon M, Jueliger S, Taverna P, Barba L, Marinari E, Pellegatta S et al (2016) Decitabine treatment of glioma-initiating cells enhances immune recognition and killing. PLoS One 11:e0162105. https://doi.org/10.1371/ journal.pone.0162105

41. Semenza GL (2012) Hypoxia-inducible factors: mediators of cancer progression and targets for cancer therapy. Trends Pharmacol Sci 33:207-214. https://doi.org/10.1016/j.tips.2012.01.005

42. Shen Y, Devgan G, Darnell JE Jr, Bromberg JF (2001) Constitutively activated Stat3 protects fibroblasts from serum withdrawal and UV-induced apoptosis and antagonizes the proapoptotic effects of activated Stat1. Proc Natl Acad Sci U S A 98:1543-1548. https://doi.org/10.1073/pnas.041588198

43. Soeda A, Park M, Lee D, Mintz A, Androutsellis-Theotokis A, McKay RD, Engh J, Iwama T, Kunisada T, Kassam AB et al (2009) Hypoxia promotes expansion of the CD133-positive glioma stem cells through activation of HIF-1alpha. Oncogene 28:3949-3959. https://doi.org/10.1038/onc.2009.252

44. Sondergaard KL, Hilton DA, Penney M, Ollerenshaw M, Demaine AG (2002) Expression of hypoxia-inducible factor 1alpha in tumours of patients with glioblastoma. Neuropathol Appl Neurobiol 28:210-217

45. Stark GR, Darnell JE Jr (2012) The JAK-STAT pathway at twenty. Immunity 36 503-514. https://doi.org/10.1016/j.immuni.2012.03.013

46. Timofeeva OA, Chasovskikh S, Lonskaya I, Tarasova NI, Khavrutskii L, Tarasov SG, Zhang X, Korostyshevskiy VR, Cheema A, Zhang L et al (2012) Mechanisms of unphosphorylated STAT3 transcription factor binding to DNA. J Biol Chem 287: 14192-14200. https://doi.org/10.1074/jbc.M111.323899

47. Tsai HC, Li H, Van Neste L, Cai Y, Robert C, Rassool FV, Shin JJ, Harbom KM, Beaty R, Pappou E et al (2012) Transient low doses of DNA-demethylating agents exert durable antitumor effects on hematological and epithelial tumor cells. Cancer Cell 21:430-446. https://doi.org/10.1016/j.ccr.2011.12.029

48. Wegrzyn J, Potla R, Chwae YJ, Sepuri NB, Zhang Q, Koeck T, Derecka M, Szczepanek K, Szelag M, Gornicka A et al (2009) Function of mitochondrial Stat3 in cellular respiration. Science 323:793-797. https://doi.org/10.1126/ science.1164551

49. Wick W, Weller M, van den Bent M, Sanson M, Weiler M, von Deimling A, Plass C, Hegi M, Platten M, Reifenberger G (2014) MGMT testing--the challenges for biomarker-based glioma treatment. Nat Rev Neurol 10:372-385. https://doi.org/10.1038/nrneurol.2014.100

50. Woo SR, Corrales L, Gajewski TF (2015) Innate immune recognition of cancer. Annu Rev Immunol 33:445-474. https://doi.org/10.1146/annurevimmunol-032414-112043

51. Xi S, Dyer KF, Kimak M, Zhang Q, Gooding WE, Chaillet JR, Chai RL, Ferrell RE, Zamboni B, Hunt J et al (2006) Decreased STAT1 expression by promoter methylation in squamous cell carcinogenesis. J Natl Cancer Inst 98:181-189. https://doi.org/10.1093/jnci/djj020

52. Zagzag D, Zhong H, Scalzitti JM, Laughner E, Simons JW, Semenza GL (2000) Expression of hypoxia-inducible factor 1alpha in brain tumors: association with angiogenesis, invasion, and progression. Cancer 88:2606-2618

53. Zhang Q, Wang HY, Liu X, Wasik MA (2007) STAT5A is epigenetically silenced by the tyrosine kinase NPM1-ALK and acts as a tumor suppressor by reciprocally inhibiting NPM1-ALK expression. Nat Med 13:1341-1348. https://doi.org/10.1038/nm1659

\section{Publisher's Note}

Springer Nature remains neutral with regard to jurisdictional claims in published maps and institutional affiliations. 